ISSN 1855-3966 (printed edn.), ISSN 1855-3974 (electronic edn.)

ARS MATHEMATICA CONTEMPORANEA 20 (2021) 103-127

https://doi.org/10.26493/1855-3974.2101.b76

(Also available at http://amc-journal.eu)

\title{
On few-class $Q$-polynomial association schemes: feasible parameters and nonexistence results
}

\author{
Alexander L. Gavrilyuk * \\ Center for Math Research and Education, Pusan National University, \\ 2, Busandaehak-ro 63beon-gil, Geumjeong-gu, Busan, 46241, Republic of Korea \\ Janoš Vidali $^{\dagger}$ \\ Faculty of Mathematics and Physics, University of Ljubljana, \\ Jadranska ulica 21, 1000 Ljubljana, Slovenia, and \\ Institute of Mathematics, Physics and Mechanics, \\ Jadranska ulica 19, 1000 Ljubljana, Slovenia

\section{Jason S. Williford $\ddagger$} \\ Department of Mathematics and Statistics, University of Wyoming, \\ 1000 E. University Ave., Laramie, WY 82071, United States of America
}

Received 28 August 2019, accepted 28 August 2020, published online 19 August 2021

\begin{abstract}
We present the tables of feasible parameters of primitive 3-class $Q$-polynomial association schemes and 4- and 5-class $Q$-bipartite association schemes (on up to 2800, 10000, and 50000 vertices, respectively), accompanied by a number of nonexistence results for such schemes obtained by analysing triple intersection numbers of putative open cases.

Keywords: Association scheme, Q-polynomial, feasible parameters, distance-regular graph.

Math. Subj. Class. (2020): 05E30

* The author is supported by BK21plus Center for Math Research and Education at Pusan National University, by Basic Science Research Program through the National Research Foundation of Korea (NRF) funded by the Ministry of Education (grant number NRF-2018R1D1A1B07047427) and by the Slovenian Research Agency (Slovenia-Russia bilateral grant number BI-RU/19-20-007).

${ }^{\dagger}$ The author is supported by the Slovenian Research Agency (research program P1-0285, research projects J1-8130, J1-1691, J1-1692 and Slovenia-Russia bilateral grant (number BI-RU/19-20-007)).

¥ The author was supported by National Science Foundation (NSF) grant DMS-1400281.

E-mail addresses: gavrilyuk@ riko.shimane-u.ac.jp (Alexander L. Gavrilyuk), janos.vidali@fmf.uni-lj.si (Janoš Vidali), jwillif1@uwyo.edu (Jason S. Williford)
\end{abstract}

()(7) This work is licensed under https://creativecommons.org/licenses/by/4.0/ 


\section{Introduction}

Much attention in literature on association schemes has been paid to distance-regular graphs, in particular to those of diameter 2, also known as strongly regular graphs - however, their complete classification is still a widely open problem. The tables of their feasible parameters, maintained by A. E. Brouwer [4, 5], are very helpful for the algebraic combinatorics community, in particular when one wants to check whether a certain example has already been proven (not) to exist, to be unique, etc. Compiling such a table can be a challenging problem, as, for example, some feasibility conditions require calculating roots of high degree polynomials.

The goal of this work is to present the tables of feasible parameters of $Q$-polynomial association schemes, compiled by the third author, and accompanied by a number of nonexistence results obtained by the first two authors.

Recall that $Q$-polynomial association schemes can be seen as a counterpart of distanceregular graphs, which, however, remains much less explored, although they have received considerable attention in the last few years [11, 25, 27, 28] due to their connection with some objects in quantum information theory such as equiangular lines and real mutually unbiased bases [24].

More precisely, let $A_{0}, \ldots, A_{D}$ and $E_{0}, \ldots, E_{D}$ denote the adjacency matrices and the primitive idempotents of an association scheme, respectively. An association scheme is P-polynomial (or metric) if, after suitably reordering the relations, there exist polynomials $v_{i}$ of degree $i$ such that $A_{i}=v_{i}\left(A_{1}\right)(0 \leq i \leq D)$. If this is the case, the matrix $A_{i}$ can be seen as the distance- $i$ adjacency matrix of a distance-regular graph and vice-versa. Similarly, an association scheme is Q-polynomial (or cometric) if, after suitably reordering the eigenspaces, there exist polynomials $v_{j}^{*}$ of degree $j$ such that $E_{j}=v_{j}^{*}\left(E_{1}\right)(0 \leq j \leq D)$, where the matrix multiplication is entrywise. These notions are due to Delsarte [15], who introduced the $P$-polynomial property as an algebraic definition of association schemes generated by distance-regular graphs, and then defined $Q$-polynomial association schemes as the dual concept to $P$-polynomial association schemes.

Many important examples of $P$-polynomial association schemes, which arise from classical algebraic objects such as dual polar spaces and forms over finite fields, also possess the $Q$-polynomial property. Bannai and Ito [1] posed the following conjecture.

Conjecture 1.1. For $D$ large enough, a primitive association scheme of $D$ classes is $P$ polynomial if and only if it is $Q$-polynomial.

We are not aware of any progress towards its proof. The discovery of a feasible set of parameters of hypothetical counter-examples (see [30]) casts some doubt on the conjecture, and in the very least shows that this will likely be difficult to prove (see the next section for the definition of feasible parameter sets). Moreover, the problem of classification of association schemes which are both $P$ - and $Q$-polynomial (i.e., $Q$-polynomial distanceregular graphs) is still open. We refer the reader to [13] for its current state.

Recall that, for a $P$-polynomial association scheme defined on a set $X$, its intersection numbers $p_{i j}^{k}$ satisfy the triangle inequality: $p_{i j}^{k}=0$ if $|i-j|>k$ or $i+j<k$, which naturally gives rise to a graph structure on $X$. Perhaps, due to the lack of such an intuitive combinatorial characterization, much less is known about $Q$-polynomial association schemes when the $P$-polynomial property is absent (which also indicates that there should be much more left to discover). To date, only few examples of $Q$-polynomial schemes are known which are neither $P$-polynomial nor duals of $P$-polynomial schemes [28] - most 
of them are imprimitive and related to combinatorial designs. The first infinite family of primitive $Q$-polynomial schemes that are not also $P$-polynomial was recently constructed in [31]. Due to Conjecture 1.1, it seems that the most promising area for constructing new examples of $Q$-polynomial association schemes which are not $P$-polynomial includes those with few classes, say, in the range $3 \leq D \leq 6$. The tables of feasible parameters of primitive 3-class $Q$-polynomial association schemes and 4- and 5-class $Q$-bipartite association schemes presented in Section 3 may serve as a source for new constructions.

We note that imprimitive $Q$-polynomial 3-class schemes are either Taylor graphs (see [5, pp. 4-6]) or linked systems of symmetric designs (see [27]). For current research on $Q$ antipodal 4- and 5-class association schemes, see [24, 25] and [11]. Due to this recent work on $Q$-antipodal schemes, the third author has focused only on the less studied primitive and $Q$-bipartite cases in his tables. We note the primitive case is far more computationally demanding than the $Q$-bipartite case, and this is the reason the class number in the tables does not go to 4 or 5 .

The parameters of $P$-polynomial association schemes are restricted by a number of conditions implied by the triangle inequality. On the other hand, the $Q$-polynomial property allows us to consider triple intersection numbers with respect to some triples of vertices, which can be thought of as a generalization of intersection numbers to triples of starting vertices instead of pairs. This technique has been previously used by various researchers [8, 10, 17, 21, 22, 23, 36, 37], mostly to prove nonexistence of some strongly regular and distance-regular graphs with equality in the so-called Krein conditions, in which case combining the restrictions implied by the triangle inequality with triple intersection numbers seems the most fruitful. Yet, while calculating triple intersection numbers when the $P$-polynomial property is absent is harder, we managed to rule out a number of open cases from the tables. This includes a putative $Q$-polynomial association scheme on 91 vertices whose existence has been open since 1999 [12].

The paper is organized as follows. In Section 2, we recall the basic theory of association schemes and their triple intersection numbers. In Section 3, we comment on the tables of feasible parameters of $Q$-polynomial association schemes and how they were generated. In Section 4, we explain in detail the analysis of triple intersection numbers of $Q$-polynomial association schemes and prove nonexistence for many open cases from the tables. Finally, in Section 5, we discuss the generalization of triple intersection numbers to quadruples of vertices.

\section{Preliminaries}

In this section we prepare the notions needed in subsequent sections.

\subsection{Association schemes}

Let $X$ be a finite set of vertices and $\left\{R_{0}, R_{1}, \ldots, R_{D}\right\}$ be a set of non-empty subsets of $X \times X$. Let $A_{i}$ denote the adjacency matrix of the (di-)graph $\left(X, R_{i}\right)(0 \leq i \leq D)$. The pair $\left(X,\left\{R_{i}\right\}_{i=0}^{D}\right)$ is called a (symmetric) association scheme of $D$ classes (or a $D$-class scheme for short) if the following conditions hold:

(1) $A_{0}=I_{|X|}$, which is the identity matrix of size $|X|$,

(2) $\sum_{i=0}^{D} A_{i}=J_{|X|}$, which is the square all-one matrix of size $|X|$,

(3) $A_{i}^{\top}=A_{i}(1 \leq i \leq D)$, 
(4) $A_{i} A_{j}=\sum_{k=0}^{D} p_{i j}^{k} A_{k}$, where $p_{i j}^{k}$ are nonnegative integers $(0 \leq i, j \leq D)$.

The nonnegative integers $p_{i j}^{k}$ are called intersection numbers: for a pair of vertices $x, y \in X$ with $(x, y) \in R_{k}$ and integers $i, j(0 \leq i, j, k \leq D), p_{i j}^{k}$ equals the number of vertices $z \in X$ such that $(x, z) \in R_{i},(y, z) \in R_{j}$.

The vector space $\mathcal{A}$ over $\mathbb{R}$ spanned by the matrices $A_{i}$ forms an algebra. Since $\mathcal{A}$ is commutative and semisimple, there exists a unique basis of $\mathcal{A}$ consisting of primitive idempotents $E_{0}=\frac{1}{|X|} J_{|X|}, E_{1}, \ldots, E_{D}$ (i.e., projectors onto the maximal common eigenspaces of $\left.A_{0}, \ldots, A_{D}\right)$. Since the algebra $\mathcal{A}$ is closed under the entry-wise multiplication denoted by $\circ$, we define the Krein parameters $q_{i j}^{k}(0 \leq i, j, k \leq D)$ by

$$
E_{i} \circ E_{j}=\frac{1}{|X|} \sum_{k=0}^{D} q_{i j}^{k} E_{k}
$$

It is known that the Krein parameters are nonnegative real numbers (see [15, Lemma 2.4]). Since both $\left\{A_{0}, A_{1}, \ldots, A_{D}\right\}$ and $\left\{E_{0}, E_{1}, \ldots, E_{D}\right\}$ form bases of $\mathcal{A}$, there exists matrices $P=\left(P_{i j}\right)_{i, j=0}^{D}$ and $Q=\left(Q_{i j}\right)_{i, j=0}^{D}$ defined by

$$
A_{i}=\sum_{j=0}^{D} P_{j i} E_{j} \quad \text { and } \quad E_{i}=\frac{1}{|X|} \sum_{j=0}^{D} Q_{j i} A_{j}
$$

The matrices $P$ and $Q$ are called the first and second eigenmatrix of $\left(X,\left\{R_{i}\right\}_{i=0}^{D}\right)$.

Let $n_{i}, 0 \leq i \leq D$, denote the valency of the graph $\left(X, R_{i}\right)$, and $m_{j}, 0 \leq j \leq D$, denote the multiplicity of the eigenspace of $A_{0}, \ldots, A_{D}$ corresponding to $E_{j}$. Note that $n_{i}=p_{i i}^{0}$, while $m_{j}=q_{j j}^{0}$.

For an association scheme $\left(X,\left\{R_{i}\right\}_{i=0}^{D}\right)$, an ordering of $A_{1}, \ldots, A_{D}$ such that for each $i(0 \leq i \leq D)$, there exists a polynomial $v_{i}(x)$ of degree $i$ with $P_{j i}=v_{i}\left(P_{j 1}\right)(0 \leq j \leq D)$, is called a $P$-polynomial ordering of relations. An association scheme is said to be $P$ polynomial if it admits a $P$-polynomial ordering of relations. The notion of an association scheme together with a $P$-polynomial ordering of relations is equivalent to the notion of a distance-regular graph - such a graph has adjacency matrix $A_{1}$, and $A_{i}(0 \leq i \leq D)$ is the adjacency matrix of its distance- $i$ graph (i.e., $(x, y) \in R_{i}$ precisely when $x$ and $y$ are at distance $i$ in the graph), and the number of classes equals the diameter of the graph. It is also known that an ordering of relations is $P$-polynomial if and only if the matrix of intersection numbers $L_{1}$, where $L_{i}:=\left(p_{i j}^{k}\right)_{k, j=0}^{D}(0 \leq i \leq D)$, is a tridiagonal matrix with nonzero superdiagonal and subdiagonal [1, p. 189] - then $p_{i j}^{k}=0$ holds whenever the triple $(i, j, k)$ does not satisfy the triangle inequality (i.e., when $|i-j|<k$ or $i+j>k$ ). For a $P$-polynomial ordering of relations of an association scheme, set $a_{i}=p_{1, i}^{i}, b_{i}=p_{1, i+1}^{i}$, and $c_{i}=p_{1, i-1}^{i}$. These intersection numbers are usually gathered in the intersection array $\left\{b_{0}, b_{1}, \ldots, b_{D-1} ; c_{1}, c_{2}, \ldots, c_{D}\right\}$, as the remaining intersection numbers can be computed from them (in particular, $a_{i}=b_{0}-b_{i}-c_{i}$ for all $i$, where $b_{D}=c_{0}=0$ ). For an association scheme with a $P$-polynomial ordering of relations, the ordering $E_{1}, \ldots, E_{D}$ is called the natural ordering of eigenspaces if $\left(P_{i 1}\right)_{i=0}^{D}$ is a decreasing sequence.

Dually, for an association scheme $\left(X,\left\{R_{i}\right\}_{i=0}^{D}\right)$, an ordering of $E_{1}, \ldots, E_{D}$ such that for each $i(0 \leq i \leq D)$, there exists a polynomial $v_{i}^{*}(x)$ of degree $i$ with $Q_{j i}=v_{i}^{*}\left(Q_{j 1}\right)$ $(0 \leq j \leq D)$, is called a $Q$-polynomial ordering of eigenspaces. An association scheme 
is said to be $Q$-polynomial if it admits a $Q$-polynomial ordering of eigenspaces. Similarly as before, it is known that an ordering of eigenspaces is $Q$-polynomial if and only if the matrix of Krein parameters $L_{1}^{*}$, where $L_{i}^{*}:=\left(q_{i j}^{k}\right)_{k, j=0}^{D}(0 \leq i \leq D)$, is a tridiagonal matrix with nonzero superdiagonal and subdiagonal [1, p. 193] - then $q_{i j}^{k}=0$ holds whenever the triple $(i, j, k)$ does not satisfy the triangle inequality. For a $Q$-polynomial ordering of eigenspaces, set $a_{i}^{*}=q_{1, i}^{i}, b_{i}^{*}=q_{1, i+1}^{i}$, and $c_{i}^{*}=q_{1, i-1}^{i}$. Again, these Krein parameters are usually gathered in the Krein array $\left\{b_{0}^{*}, b_{1}^{*}, \ldots, b_{D-1}^{*} ; c_{1}^{*}, c_{2}^{*}, \ldots, c_{D}^{*}\right\}$ containing all the information needed to compute the remaining Krein parameters (in particular, we have $a_{i}^{*}=b_{0}^{*}-b_{i}^{*}-c_{i}^{*}$ for all $i$, where $b_{D}^{*}=c_{0}^{*}=0$ ). For an association scheme with a $Q$ polynomial ordering of eigenspaces, the ordering $A_{1}, \ldots, A_{D}$ is called the natural ordering of relations if $\left(Q_{i 1}\right)_{i=0}^{D}$ is a decreasing sequence. Unlike for the $P$-polynomial association schemes, there is no known general combinatorial characterization of $Q$-polynomial association schemes.

An association scheme is called primitive if all of $A_{1}, \ldots, A_{D}$ are adjacency matrices of connected graphs. It is known that a distance-regular graph is imprimitive precisely when it is a cycle of composite length, an antipodal graph, or a bipartite graph (possibly more than one of these), see [5, Thm. 4.2.1]. The last two properties can be recognised from the intersection array as $b_{i}=c_{D-i}(0 \leq i \leq D, i \neq\lfloor D / 2\rfloor)$ and $a_{i}=0(0 \leq i \leq D)$, respectively. We may define dual properties for a $Q$-polynomial association scheme - we say that it is $Q$-antipodal if $b_{i}^{*}=c_{D-i}^{*}(0 \leq i \leq D, i \neq\lfloor D / 2\rfloor)$, and $Q$-bipartite if $a_{i}^{*}=0(0 \leq i \leq D)$. All imprimitive $Q$-polynomial association schemes are schemes of cycles of composite length, $Q$-antipodal or $Q$-bipartite (again, possibly more than one of these). The original classification theorem by Suzuki [34] allowed two more cases, which have however been ruled out later $[9,35]$. An association scheme that is both $P$ - and $Q$ polynomial is $Q$-antipodal if and only if it is bipartite, and is $Q$-bipartite if and only if it is antipodal.

A formal dual of an association scheme with first and second eigenmatrices $P$ and $Q$ is an association scheme such that, for some orderings of its relations and eigenspaces, its first and second eigenmatrices are $Q$ and $P$, respectively. Note that this duality occurs on the level of parameters - an association scheme might have several formal duals, or none at all (we can speak of duality when there exists a regular abelian group of automorphisms, see [5, §2.10B]). An association scheme with $P=Q$ for some orderings of its relations and eigenspaces is called formally self-dual. For such orderings, $p_{i j}^{k}=q_{i j}^{k}(0 \leq i, j, k \leq D)$ holds - in particular, a formally self-dual association scheme is $P$-polynomial if and only if it is $Q$-polynomial, and then its intersection array matches its Krein array.

Any imprimitive association scheme with two classes is both $P$ - and $Q$-polynomial for either of the two orderings of relations and eigenspaces. The graph with adjacency matrix $A_{1}$ of such a scheme is said to be strongly regular (an SRG for short) with parameters $(n, k, \lambda, \mu)$, where $n=|X|$ is the number of vertices, $k=p_{11}^{0}$ is the valency of each vertex, and each two distinct vertices have precisely $\lambda=p_{11}^{1}$ common neighbours if they are adjacent, and $\mu=p_{11}^{2}$ common neighbours if they are not adjacent. In the sequel, we will identify $P$-polynomial association schemes with their corresponding strongly regular or distance-regular graphs.

By a parameter set of an association scheme, we mean the full set of $p_{i j}^{k}, q_{i j}^{k}, P_{i j}$ and $Q_{i j}$ described in this section, which are real numbers satisfying the identities in [5, Lemma 2.2.1, Lemma 2.3.1]. We say that a parameter set for an association scheme is feasible if it passes all known conditions for the existence of a corresponding association 
scheme. For distance-regular graphs, there are many known feasibility conditions, see [5, $13,37]$. For $Q$-polynomial association schemes, much less is known - see Section 3 for the feasibility conditions we have used.

\subsection{Triple intersection numbers}

For a triple of vertices $x, y, z \in X$ and integers $i, j, k(0 \leq i, j, k \leq D)$ we denote by $\left[\begin{array}{ccc}x & y & z \\ i & j & k\end{array}\right]$ (or simply $\left[\begin{array}{lll}i & j & k\end{array}\right]$ when it is clear which triple $(x, y, z)$ we have in mind) the number of vertices $w \in X$ such that $(x, w) \in R_{i},(y, w) \in R_{j}$ and $(z, w) \in R_{k}$. We call these numbers triple intersection numbers.

Unlike the intersection numbers, the triple intersection numbers depend, in general, on the particular choice of $(x, y, z)$. Nevertheless, for a fixed triple $(x, y, z)$, we may write down a system of $3 D^{2}$ linear Diophantine equations with $D^{3}$ triple intersection numbers as variables taking nonnegative values, thus relating them to the intersection numbers, cf. [22]:

$$
\sum_{\ell=0}^{D}[\ell j k]=p_{j k}^{t}, \quad \sum_{\ell=0}^{D}[i \ell k]=p_{i k}^{s}, \quad \sum_{\ell=0}^{D}[i j \ell]=p_{i j}^{r}, \quad(1 \leq i, j, k \leq D)
$$

where $(x, y) \in R_{r},(x, z) \in R_{s},(y, z) \in R_{t}$, and

$$
[0 j k]=\delta_{j r} \delta_{k s}, \quad[i \quad 0 k]=\delta_{i r} \delta_{k t}, \quad[i j 0]=\delta_{i s} \delta_{j t} \quad(0 \leq i, j, k \leq D)
$$

are constants. Note that the equations (2.3) are not all linearly independent, so the system is underdetermined in general when $D \geq 3$. Moreover, the following theorem sometimes gives additional equations.

Theorem 2.1 ([10, Theorem 3], cf. [7], [5, Theorem 2.3.2]). Let $\left(X,\left\{R_{i}\right\}_{i=0}^{D}\right)$ be an association scheme of $D$ classes with second eigenmatrix $Q$ and Krein parameters $q_{r s}^{t}$ $(0 \leq r, s, t \leq D)$. Then,

$$
q_{r s}^{t}=0 \Longleftrightarrow \sum_{i, j, k=0}^{D} Q_{i r} Q_{j s} Q_{k t}\left[\begin{array}{ccc}
x & y & z \\
i & j & k
\end{array}\right]=0 \quad \text { for all } x, y, z \in X
$$

Note that in a $Q$-polynomial association scheme, many Krein parameters are zero, and we can use Theorem 2.1 to obtain an equation for each of them.

\section{Tables of feasible parameters for $Q$-polynomial association schemes}

In this section we will describe the tables of feasible parameter sets for primitive 3-class $Q$-polynomial schemes and 4 - and 5 -class $Q$-bipartite schemes.

These tables were all completed using the MAGMA programming language (see [2]). Any parameter set meeting the following conditions was included in the table:

(1) The parameters satisfy the $Q$-polynomial condition.

(2) All $p_{i j}^{k}$ are nonnegative integers, all valencies $p_{j j}^{0}$ are positive.

(3) For each $j>0$ we have $n p_{j j}^{0}$ is even (the handshaking lemma applied to the graph $\left.\left(X, R_{j}\right)\right)$. 
(4) For each $j, k>0$ we have $p_{j j}^{0} p_{j k}^{j}$ is even (the handshaking lemma applied to the subconstituent $\left(Y,\left\{(y, z) \in Y \times Y \mid(y, z) \in R_{k}\right\}\right)$, where $x \in X$ and $Y=\{y \in$ $\left.\left.X \mid(x, y) \in R_{j}\right\}\right)$.

(5) For each $j>0$ we have $n p_{j j}^{0} p_{j j}^{j}$ is divisible by 6 (the number of triangles in each graph $\left(X, R_{j}\right)$ is integral).

(6) All $q_{i j}^{k}$ are nonnegative and for each $j$ the multiplicity $q_{j j}^{0}$ (i.e., the dimension of the $E_{j}$-eigenspace) is a positive integer (see [5, Proposition 2.2.2]).

(7) For all $i, j$ we have $\sum_{q_{i j}^{k} \neq 0} m_{k} \leq m_{i} m_{j}$ if $i \neq j$ and $\sum_{q_{i i}^{k} \neq 0} m_{k} \leq \frac{m_{i}\left(m_{i}-1\right)}{2}$ (the absolute bound, see [5, Theorem 2.3.3] and the references therein).

(8) The splitting field is at most a degree 2 extension of the rationals (see [29]).

We note that there are many other conditions known for the special case of distanceregular graphs. It was decided to apply these conditions after the construction of the table, and those not meeting these extra conditions were labelled as nonexistent with a note as to the condition not met. We leave as an open question whether if any of these conditions could be generalized to any cases beyond distance-regular graphs; this (perhaps faint) hope is the main reason that they are included in the table.

We begin with the tables for $Q$-bipartite schemes, since this case is somewhat simpler than the primitive case. Schemes which are $Q$-bipartite are formally dual to bipartite distance-regular graphs. As a consequence, the formal dual to [5, Theorem 4.2.2(i)] gives the Krein array for the quotient scheme of a $Q$-bipartite scheme (see [27]). Namely, if the scheme has Krein array $\left\{b_{0}^{*}, b_{1}^{*}, \ldots, b_{D-1}^{*} ; c_{1}^{*}, \ldots, c_{D}^{*}\right\}$ and $q_{11}^{2}=\mu^{*}$, then the Krein array of the quotient is

$$
\left\{\frac{b_{0}^{*} b_{1}^{*}}{\mu^{*}}, \frac{b_{2}^{*} b_{3}^{*}}{\mu^{*}}, \ldots, \frac{b_{2 t-2}^{*} b_{2 t-1}^{*}}{\mu^{*}} ; \frac{c_{1}^{*} c_{2}^{*}}{\mu^{*}}, \frac{c_{3}^{*} c_{4}^{*}}{\mu^{*}}, \ldots, \frac{c_{2 t-1}^{*} c_{2 t}^{*}}{\mu^{*}}\right\},
$$

where $t=\left\lfloor\frac{D}{2}\right\rfloor$. Note that the quotient scheme has multiplicities $1, m_{2}, m_{4}, \ldots, m_{2 t}$, from which it follows that the condition $\sum_{i=0}^{t} m_{2 i}=\sum_{i=1}^{D-t} m_{2 i-1}$ must be satisfied for a $D$-class $Q$-bipartite scheme.

When $D=4,5$ we obtain $t=2$, so the quotient structure is a strongly regular graph. A database of strongly regular graph parameters up to 5000 vertices can be generated very quickly. From there, we can use the above condition on the multiplicities. The following proposition shows that the multiplicities determine all the parameters of the scheme.

Proposition 3.1. A D-class $Q$-bipartite $Q$-polynomial association scheme with $D \in\{4,5\}$ and multiplicities $1, m_{1}, m_{2}, \ldots, m_{D}$ has the Krein array

$$
\begin{gathered}
\left\{m_{1}, m_{1}-1, \frac{m_{1}\left(m_{2}-m_{1}+1\right)}{m_{2}}, \frac{m_{1}\left(m_{3}-m_{2}+m_{1}-1\right)}{m_{3}}\right. \\
\left.1, \frac{m_{1}\left(m_{1}-1\right)}{m_{2}}, \frac{m_{1}\left(m_{2}-m_{1}+1\right)}{m_{3}}, m_{1}\right\}
\end{gathered}
$$

or

$$
\begin{gathered}
\left\{m_{1}, m_{1}-1, \frac{m_{1}\left(m_{2}-m_{1}+1\right)}{m_{2}}, \frac{m_{1}\left(m_{3}-m_{2}+m_{1}-1\right)}{m_{3}}, \frac{m_{1}\left(m_{4}-m_{3}+m_{2}-m_{1}+1\right)}{m_{4}}\right. \\
\left.1, \frac{m_{1}\left(m_{1}-1\right)}{m_{2}}, \frac{m_{1}\left(m_{2}-m_{1}+1\right)}{m_{3}}, \frac{m_{1}\left(m_{3}-m_{2}+m_{1}-1\right)}{m_{4}}, m_{1}\right\}
\end{gathered}
$$


Proof. Follows easily from the identities of [5, Lemma 2.3.1].

In the 4-class case, the parameters are entirely determined by the quotient's multiplicities (with a chosen $Q$-polynomial ordering) and $m_{1}$. To search, we take a strongly regular graph parameter set, choose one of two possible orderings for its multiplicities, calling its multiplicities $m_{0}=1, m_{2}, m_{4}$. From the absolute bound, we have $1+m_{2} \leq \frac{m_{1}\left(m_{1}+1\right)}{2}$, and from the positivity of $c_{2}^{*}$ we have $\frac{\left(m_{2}-m_{1}+1\right) m_{1}}{m_{2}} \geq 0$. We then search over all $\sqrt{2\left(1+m_{2}\right)}-\frac{1}{2} \leq m_{1} \leq m_{2}$, checking the conditions above. Given that we are iterating over SRG parameters together with two orderings and one integer, this search is very fast. The limitation of the table to 10000 vertices is mainly readability and practicality. The third author has unpublished tables (without comments or details) to 100000 vertices.

We note that $Q$-bipartite schemes with 5 classes are very similar, except we must iterate over both $m_{1}$ and $m_{3}$. Again, this is a very quick search, but the relative scarcity of 5-class parameter sets makes listing up to 50000 vertices, with annotation, manageable. The table actually goes slightly higher, to 50520 vertices, because of the existence of an example on that number of vertices.

The trickiest search was the primitive 3 -class $Q$-polynomial parameter sets. In this case, there is no non-trivial quotient scheme to build on.

We use the following observation.

Theorem 3.2. A primitive Q-polynomial association scheme of 3 classes must have a matrix $L_{i}$ with 4 distinct eigenvalues.

Proof. Assume not. If a matrix $A_{i}$ has only two distinct eigenvalues, it is either complete, contradicting the fact that it is a 3-class scheme, or a disjoint union of more than one complete graph, contradicting the fact the scheme is primitive. Therefore, the only case left to consider is when $A_{1}, A_{2}, A_{3}$ all have three distinct eigenvalues, meaning the graphs are all strongly regular. A 3-class scheme where every non-trivial relation is strongly-regular is amorphic, see [19] and [14] for a definition and details on amorphic schemes. It was shown in [20] that amorphic schemes are formally self-dual. This implies that no column of $Q$ has 4 distinct entries. Therefore, the second eigenmatrix $Q$ cannot be generated by one column via polynomials, thus the scheme cannot be $Q$-polynomial.

We note that, in fact, all $Q$-polynomial $D$-class schemes must have a relation with $D+1$ distinct eigenvalues. However, the above theorem and its proof is sufficient for our needs.

From this we conclude that each 3 -class primitive $Q$-polynomial scheme has an adjacency matrix, which we label $A_{1}$, which has four distinct eigenvalues. Then the corresponding $4 \times 4$ intersection matrix $L_{1}$ has four distinct eigenvalues. From this matrix, all of the other parameters may be determined. In particular, from [5, Proposition 2.2.2], the left-eigenvectors of $L_{1}$, normalized so their leftmost entry is 1 , must be the rows of $P$.

The rest of the parameters can be derived from the equations:

$$
\begin{aligned}
L_{j} & =P^{-1} \operatorname{diag}\left(P_{0 j}, P_{1 j}, \ldots, P_{D j}\right) P, \\
L_{j}^{*} & =Q^{-1} \operatorname{diag}\left(Q_{0 j}, Q_{1 j}, \ldots, Q_{D j}\right) Q .
\end{aligned}
$$

However, checking the $Q$-polynomial condition is done before the computation of all parameters. We use the following theorem, a proof of which can be found in [30]. 
Theorem 3.3. Let $L_{i}$ be an intersection matrix of a D-class association scheme, where $L_{i}$ has exactly $D+1$ distinct eigenvalues. Then the scheme is $Q$-polynomial if and only if there is a Vandermonde matrix $U$ such that $U^{-1} L_{i} U=T$ where $T$ is upper triangular.

It is not hard to show that, without loss of generality, we can take $T_{01}$ to be 0 , implying that the first column of $U$ is an eigenvector of $L_{1}$. We only then need to iterate over the three (nontrivial) eigenvectors of $L_{1}$ to check this condition. If the $Q$-polynomial condition is met, the rest of the parameters are computed and checked for the above conditions.

The schemes are then split into types depending on whether there is a strongly regular graph as a relation, and whether the splitting field is rational or not. These are split in this manner to aid in computation (following the list of types we give details on how these were used):

(1) Diameter 3 distance-regular graphs (DRG for short).

(2) No diameter $3 \mathrm{DRG}$, there is a strongly regular graph as a relation, the splitting field is the rational field.

(3) No diameter 3 DRG, there is a strongly regular graph as a relation, the splitting field is a degree- 2 extension of the rational field.

(4) No diameter $3 \mathrm{DRG}$, there is no strongly regular graph as a relation, the splitting field is the rational field.

(5) No diameter $3 \mathrm{DRG}$, there is no strongly regular graph as a relation, the splitting field is a degree- 2 extension of the rational field.

We note that we do not have any examples of primitive 3-class $Q$-polynomial schemes with an irrational splitting field, but there are open parameter sets of such (for example, see entry $\langle 216,20\rangle$ in the third author's primitive 3-class table at [39]). It would be interesting to determine if these exist. We also point out that all the feasible parameter sets known to us have rational Krein parameters.

Type 1. For DRG's, we iterated over the number of vertices, intersection array and valencies. The order was $n, b_{0}=n_{1}, b_{1}, n_{2}$ (noting $n_{2}$ is a divisor of $n_{1} b_{1}$ ), then $b_{2}$ (noting $b_{2}$ must be a multiple of $\frac{n_{3}}{\operatorname{gcd}\left(n_{2}, n_{3}\right)}$, where $\left.n_{3}=n-n_{1}-n_{2}\right)$, from which the rest could be determined.

When there is no DRG, it is tempting to try to formally dualize the above process. However, the Krein parameters of a scheme do not have to be integral, or even rational. For this reason, it seemed more advantageous to iterate over parameters that needed to be integral, namely the parameters $p_{i j}^{k}$. All arithmetic was done in MAGMA using the rational field, or a splitting field of a degree two irreducible polynomial over the rationals. Floating point arithmetic was avoided to minimize numerical errors.

For the rest of the types, $L_{1}$ and the valencies were iterated over. In particular, the parameters $a=p_{12}^{1}, b=p_{13}^{1}$ and $c=p_{13}^{2}$, together with $n, n_{1}, n_{2}$ determine the rest of $L_{1}$, noting that $a+b \leq n_{1}-1$ and $c \leq n_{1}-\frac{n_{1} a}{n_{2}}$. Any matrix without 4 distinct eigenvalues or with an irreducible cubic factor in its characteristic polynomial was discarded.

Types 2 and 3. For these types, we iterate over strongly regular graphs first, with parameters $(n, k, \lambda, \mu)$. We choose $A_{3}$ to be the adjacency matrix of the strongly regular graph relation, and $L_{1}, L_{2}$ to be fissions of the complement. Given this, the choice of $n_{1}$ will 
determine $n_{2}$. The possibilities for $n_{1}$ can be narrowed by observing that $p_{33}^{1}=\mu, n_{3}=k$ and $p_{33}^{1} n_{1}=p_{13}^{3} n_{3}$, implying that $n_{1}$ is divisible by $\frac{n_{3}}{\operatorname{gcd}\left(n_{3}, \mu\right)}$.

Using similar identities, we find $b$ is divisible by $\frac{n_{3}}{\operatorname{gcd}\left(n_{1}, n_{3}\right)}, a$ is divisible by $\frac{n_{2}}{\operatorname{gcd}\left(n_{1}, n_{2}\right)}$, and $c=\frac{n_{1}\left(n_{3}-b-\mu\right)}{n_{2}}$. After choosing these parameters all of $L_{1}$ follows.

Types 4 and 5. For these types, we know $L_{1}, L_{2}$ and $L_{3}$ all have 4 distinct eigenvalues. Therefore, we can assume $n_{1}$ is the smallest valency, and that $a \leq b$. Using $a$ is divisible by $\frac{n_{2}}{\operatorname{gcd}\left(n_{1}, n_{2}\right)}, b$ is divisible by $\frac{n_{3}}{\operatorname{gcd}\left(n_{1}, n_{3}\right)}$, and $n_{2}$ divides $a n_{1}$, we choose $n_{1}, a, n_{2}, b, c$, from which the rest is determined. This is the slowest part of the search, and the reason the primitive table goes to 2800 vertices.

We close this section with some comments on the irrational splitting field types. The 2-class primitive $Q$-polynomial association schemes are equivalent to complementary pairs of primitive strongly regular graphs. The only case where strongly regular graphs have an irrational splitting field is the so-called "half-case", when the graph has valency $\frac{n-1}{2}$. Such graphs do exist, for example the Paley graphs for non-square prime powers $q$ with $q \equiv 1$ $(\bmod 4)$. We note that no primitive $Q$-polynomial schemes with more than 2 classes and a quadratic splitting field are known. All feasible parameter sets we know of are 3-class and have a strongly regular graph relation (type 3 ). The corresponding strongly regular graphs are also all unknown (see [4]). We have no feasible parameter set for type 5. However, one type 5 parameter set satisfied all criteria except the handshaking lemma. Given this, we expect feasible parameter sets for type 5 to exist, but may be quite large. This parameter set is listed below (including $L_{1}^{*}$, so it can be seen it is $Q$-polynomial, but not including the other $L_{i}^{*}$ matrices), though this set is not included in the online table:

$$
\begin{aligned}
& P=\left(\begin{array}{cccc}
1 & 285 & 285 & 405 \\
1 & 19+8 \sqrt{19} & -38+1 \sqrt{19} & 18-9 \sqrt{19} \\
1 & -3 & 5 & -3 \\
1 & 19-8 \sqrt{19} & -38-1 \sqrt{19} & 18+9 \sqrt{19}
\end{array}\right), Q=\left(\begin{array}{cccc}
1 & 60 & 855 & 60 \\
1 & \frac{76+32 \sqrt{19}}{19} & -9 & \frac{76-32 \sqrt{19}}{19} \\
1 & \frac{-152+4 \sqrt{19}}{19} & 15 & \frac{-152-4 \sqrt{19}}{19} \\
1 & \frac{8-4 \sqrt{19}}{3} & \frac{-19}{3} & \frac{8+4 \sqrt{19}}{3}
\end{array}\right), \\
& L_{1}=\left(\begin{array}{cccc}
0 & 285 & 0 & 0 \\
1 & 116 & 60 & 108 \\
0 & 60 & 90 & 135 \\
0 & 76 & 95 & 114
\end{array}\right), \quad L_{2}=\left(\begin{array}{cccc}
0 & 0 & 285 & 0 \\
0 & 60 & 90 & 135 \\
1 & 90 & 59 & 135 \\
0 & 95 & 95 & 95
\end{array}\right), \quad L_{3}=\left(\begin{array}{cccc}
0 & 0 & 0 & 405 \\
0 & 108 & 135 & 162 \\
0 & 135 & 135 & 135 \\
1 & 114 & 95 & 195
\end{array}\right), \\
& L_{1}^{*}=\left(\begin{array}{cccc}
0 & 60 & 0 & 0 \\
1 & \frac{400+32 \sqrt{19}}{61} & \frac{3199-32 \sqrt{19}}{61} & 0 \\
0 & \frac{12796-128 \sqrt{19}}{3477} & \frac{181184+128 \sqrt{19}}{3477} & \frac{80}{19} \\
0 & 0 & 60 & 0
\end{array}\right) .
\end{aligned}
$$

While feasible parameters may exist, the complete lack of examples elicits the following question:

Question 3.4. Do all 3-class primitive $Q$-polynomial schemes have a rational splitting field?

This is a special case of the so-called "Sensible Caveman" conjecture of William J. Martin:

Conjecture 3.5. For Q-polynomial schemes of 3 or more classes that is not a polygon, if the scheme is primitive then its splitting field is rational. 


\section{Nonexistence results}

We derived our nonexistence results by analyzing triple intersection numbers of $Q$-polynomial association schemes. For some choice of relations $R_{r}, R_{s}, R_{t}$, the system of Diophantine equations derived from (2.3) and Theorem 2.1 may have multiple nonnegative solutions, each giving the possible values of the triple intersection numbers with respect to a triple $(x, y, z)$ with $(x, y) \in R_{r},(x, z) \in R_{s}$ and $(y, z) \in R_{t}$. However, in certain cases, there might be no nonnegative solutions - in this case, we may conclude that an association scheme with the given parameters does not exist.

Even when there are solutions for all choices of $R_{r}, R_{s}, R_{t}$ such that $p_{r s}^{t} \neq 0$, sometimes nonexistence can be derived by other means. We may, for example, employ double counting.

Proposition 4.1. Let $x$ and $y$ be vertices of an association scheme with $(x, y) \in R_{r}$. Suppose that $\alpha_{1}, \alpha_{2}, \ldots, \alpha_{m}$ are distinct integers such that there are precisely $\kappa_{\ell}$ vertices $z$ with $(x, z) \in R_{s},(y, z) \in R_{t}$ and $\left[\begin{array}{ccc}x & y & z \\ i & j & k\end{array}\right]=\alpha_{\ell}\left(1 \leq \ell \leq m, \sum_{\ell=1}^{m} \kappa_{\ell}=p_{s t}^{r}\right)$, and $\beta_{1}, \beta_{2}, \ldots, \beta_{n}$ are distinct integers such that there are precisely $\lambda_{\ell}$ vertices $w$ with $(w, x) \in R_{i},(w, y) \in R_{j}$ and $\left[\begin{array}{lll}w & x & y \\ k & s & t\end{array}\right]=\beta_{\ell}\left(1 \leq \ell \leq n, \sum_{\ell=1}^{n} \lambda_{\ell}=p_{i j}^{r}\right)$. Then,

$$
\sum_{\ell=1}^{m} \kappa_{\ell} \alpha_{\ell}=\sum_{\ell=1}^{n} \lambda_{\ell} \beta_{\ell} .
$$

Proof. Count the number of pairs $(w, z)$ with $(x, z) \in R_{s},(y, z) \in R_{t},(w, x) \in R_{i}$, $(w, y) \in R_{j}$ and $(w, z) \in R_{k}$.

We consider the special case of Proposition 4.1 when a triple intersection number is zero for all triples of vertices in some given relations.

Corollary 4.2. Suppose that for all vertices $x, y, z$ of an association scheme with $(x, y) \in$ $R_{r},(x, z) \in R_{s},(y, z) \in R_{t},\left[\begin{array}{lll}x & y & z \\ i & j & k\end{array}\right]=0$ holds. Then, $\left[\begin{array}{lll}w & x & y \\ k & s & t\end{array}\right]=0$ holds for all vertices $w, x, y$ with $(w, x) \in R_{i},(w, y) \in R_{j}$ and $(x, y) \in R_{r}$.

Proof. Apply Proposition 4.1 to all $(x, y) \in R_{r}$, with $m \leq 1$ and $\alpha_{1}=0$. Since $\beta_{\ell}$ and $\lambda_{\ell}$ $(1 \leq \ell \leq n)$ must be nonnegative, it follows that $n \leq 1$ and $\beta_{1}=0$.

\subsection{Computer search}

The sage-drg package $[38,37]$ by the second author for the SageMath computer algebra system [32] has been used to perform computations of triple intersection numbers of $Q$-polynomial association schemes with Krein arrays that were marked as open in the tables of feasible parameter sets by the third author [39], see Section 3. The package was originally developed for the purposes of feasibility checking for intersection arrays of distance-regular graphs and included a routine to find general solutions to the system of equations for computing triple intersection numbers.

For the purposes of the current research, the package has been extended to support parameters of general association schemes, in particular, given as Krein arrays of $Q$-polynomial association schemes. Additionally, the package now supports generating integral solutions for systems of equations with constraints on the solutions (e.g., nonnegativity of 
triple intersection numbers) - these can also be added on-the-fly. The routine uses SageMath's mixed integer linear programming facilities, which support multiple solvers. We have used SageMath's default GLPK solver [26] and the CBC solver [16] in our computations - however, other solvers can also be used if they are available.

We have thus been able to implement an algorithm which tries to narrow down the possible solutions of the systems of equations for determining triple intersection numbers of an association scheme such that they satisfy Corollary 4.2, and conclude inequality if any of the systems of equations has no such feasible solutions.

(1) For each triple of relations $\left(R_{r}, R_{s}, R_{t}\right)$ such that $p_{r s}^{t}>0$, initialize an empty set of solutions, obtain a general (i.e., parametric) solution to the system of equations derived from (2.3) and Theorem 2.1, and initialize a generator of solutions with the constraint that the intersection numbers be integral and nonnegative. All generators $(r, s, t)$ are initially marked as active, and all triple intersection numbers $(r, s, t ; i, j, k)$ (representing $\left[\begin{array}{lll}x & y & z \\ i & j & k\end{array}\right]$ with $(x, y) \in R_{r},(x, z) \in R_{s}$ and $\left.(y, z) \in R_{t}\right)$ are initially marked as unknown.

(2) For each active generator, generate one solution and add it to the corresponding set of solutions. If a generator does not return a new solution (i.e., it has exhausted all of them), then mark it as inactive.

(3) For each inactive generator, verify that the corresponding set of solutions is nonempty - otherwise, terminate and conclude nonexistence.

(4) Initialize an empty set $Z$.

(5) For each unknown triple intersection number $(r, s, t ; i, j, k)$, mark it as nonzero if a solution has been found in which its value is not zero. If such a solution has not been found yet, make a copy of the generator $(r, s, t)$ with the constraint that $(r, s, t ; i, j, k)$ be nonzero, and generate one solution. If such a solution exists, add it to the set of solutions and mark $(r, s, t ; i, j, k)$ as nonzero, otherwise mark $(r, s, t ; i, j, k)$ as zero and add it to $Z$.

(6) If $Z$ is empty, terminate without concluding nonexistence.

(7) For each triple intersection number $(r, s, t ; i, j, k) \in Z$ and for each nonzero $(a, b, c$; $d, e, f) \in\{(r, i, j ; s, t, k),(s, i, k ; r, t, j),(t, j, k ; r, s, i)\}$, remove all solutions from the corresponding set in which the value of the latter is nonzero, mark $(a, b, c ; d, e, f)$ as zero, mark all nonzero $(a, b, c ; \ell, m, n)$ with $(\ell, m, n) \neq(d, e, f)$ as unknown, and add a constraint that $(a, b, c ; d, e, f)$ be zero to the generator $(a, b, c)$ if it is active.

(8) Go to (2).

Note that generators and triple intersection numbers are considered equivalent under permutation of vertices, i.e., under actions $(r, s, t) \mapsto(r, s, t)^{\pi}$ and $(r, s, t ; i, j, k) \mapsto$ $\left((r, s, t)^{\pi} ;(i, j, k)^{\pi^{(13)}}\right)$ for $\pi \in S_{3}$.

The above algorithm is available as the check_quadruples method of sage-drg's ASParameters class. We ran it for all open cases in the tables from Section 3, and obtained 29 nonexistence results for primitive 3-class schemes, 92 nonexistence results for $Q$-bipartite 4-class schemes, and 11 nonexistence results for $Q$-bipartite 5 -class schemes. The results are summarized in the following theorem and in the tables in Appendix A. 
Theorem 4.3. A Q-polynomial association scheme with Krein array listed in one of Tables 1,2 and 3 does not exist.

Proof. In all but two cases, it suffices to observe that for some triple of relations $R_{r}, R_{s}, R_{t}$, the system of equations derived from (2.3) and Theorem 2.1 has no integral nonnegative solutions - Tables 1 and 2 list the triple $(r, s, t)$, while for all examples in Table 3 , this is true for $(r, s, t)=(1,1,1)$. Note that the natural ordering of the relations is used.

Let us now consider the cases $\langle 225,24\rangle$ and $\langle 1470,104\rangle$ from Table 1 . In the first case, the Krein array is $\{24,20,36 / 11 ; 1,30 / 11,24\}$. Such an association scheme has two $Q$ polynomial orderings, so we can augment the system of equations (2.3) with six equations derived from Theorem 2.1. Let $w, x, y, z$ be vertices such that $(x, z),(y, z) \in R_{1}$ and $(w, x),(w, y),(x, y) \in R_{3}$. Since $p_{11}^{3}=22$ and $p_{33}^{3}=3$, such vertices must exist. We first compute the triple intersection numbers with respect to $x, y, z$. There are two integral nonnegative solutions, both having $\left[\begin{array}{lll}3 & 3 & 1\end{array}\right]=0$. On the other hand, there is a single solution for the triple intersection numbers with respect to $w, x, y$, giving $\left[\begin{array}{lll}1 & 1 & 1\end{array}\right]=3$. However, this contradicts Corollary 4.2, so such an association scheme does not exist.

In the second case, the Krein array is $\{104,70,25 ; 1,7,80\}$. Let $w, x, y, z$ be vertices such that $(x, y),(x, z) \in R_{1},(w, y),(y, z) \in R_{2}$ and $(w, x) \in R_{3}$. Since $p_{12}^{1}=70$ and $p_{32}^{1}=250$, such vertices must exist. There is a single solution for the triple intersection numbers with respect to $x, y, z$, giving $\left[\begin{array}{lll}3 & 2 & 3\end{array}\right]=0$. On the other hand, there are four solutions for the triple intersection numbers with respect to $w, x, y$, from which we obtain $\left[\begin{array}{lll}3 & 1 & 2\end{array}\right] \in\{15,16,17,18\}$. Again, this contradicts Corollary 4.2, so such an association scheme does not exist. This completes the proof.

Remark 4.4. The sage-drg package repository provides two Jupyter notebooks containing the computation details in the proofs of nonexistence of two cases from Table 1:

- QPOIY-24-20-36_11-1-30_11-24. ipynb for the case $\langle 225,24\rangle$, and

- DRG-104-70-25-1-7-80. ipynb for the case $\langle 1470,104\rangle$.

Remark 4.5. The parameter set $\langle 91,12\rangle$ from Table 1 was listed by Van Dam [12] as the smallest feasible $Q$-polynomial parameter set for which no scheme is known. The next such open case is now the Krein array $\{14,108 / 11,15 / 4 ; 1,24 / 11,45 / 4\}$ for a primitive 3 -class $Q$-polynomial association scheme with 99 vertices, which was also listed by Van Dam.

Since some of the parameters from Table 1 also admit a $P$-polynomial ordering, we can derive nonexistence of distance-regular graphs with certain intersection arrays. We have also found an intersection array for a primitive $Q$-polynomial distance-regular graph of diameter 4, which is listed in [5] and [3], and for which, to the best of our knowledge, nonexistence has not been previously known.

Theorem 4.6. There is no distance-regular graph with intersection array

$$
\begin{gathered}
\\
\{83,54,21 ; 1,6,63\}, \\
\{104,70,25 ; 1,7,80\}, \\
\{195,160,28 ; 1,20,168\}, \\
\{125,108,24 ; 1,9,75\}, \\
\{126,90,10 ; 1,6,105\}, \text { or } \\
\{203,160,34 ; 1,16,170\} .
\end{gathered}
$$


Proof. The cases $\langle 1080,83\rangle,\langle 1470,104\rangle,\langle 2016,195\rangle$ and $\langle 2640,203\rangle$ from Table 1 are formally self-dual for the natural ordering of relations, while $\langle 2197,126\rangle$ is formally selfdual with ordering of relations $A_{2}, A_{3}, A_{1}$ relative to the natural ordering. In each case, the corresponding association scheme is $P$-polynomial with intersection array equal to the Krein array. The case $\langle 2106,65\rangle$ is not formally self-dual, yet the natural ordering of relations is $P$-polynomial with intersection array $\{125,108,24 ; 1,9,75\}$. In all of the above cases, Theorem 4.3 implies nonexistence of the corresponding association scheme, so a distance-regular graph with such an intersection array does not exist.

Theorem 4.7. There is no distance-regular graph with intersection array

$$
\{53,40,28,16 ; 1,4,10,28\} \text {. }
$$

Proof. Consider a distance-regular graph with intersection array $\{53,40,28,16 ; 1,4,10$, $28\}$. Such a graph is formally self-dual for the natural ordering of eigenspaces and therefore also $Q$-polynomial. Augmenting the system of equations (2.3) with twelve equations derived from Theorem 2.1 gives a two parameter solution for triple intersection numbers with respect to three vertices mutually at distances $1,3,3$. However, it turns out that there is no integral solution, leading to nonexistence of the graph.

Remark 4.8. The non-existence of a distance-regular graph with intersection array $\{53,40$, $28,16 ; 1,4,10,28\}$ also follows by applying the Terwilliger polynomial [17]. Recall that this polynomial, say $T_{\Gamma}(x)$, which depends only on the intersection numbers of a $Q$ polynomial distance-regular graph $\Gamma$ and its $Q$-polynomial ordering, satisfies:

$$
T_{\Gamma}(\eta) \geq 0
$$

where $\eta$ is any non-principal eigenvalue of the local graph of an arbitrary vertex $x$ of $\Gamma$. Furthermore, by [5, Theorem 4.4.3(i)], $\eta$ satisfies

$$
-1-\frac{b_{1}}{\theta_{1}+1} \leq \eta \leq-1-\frac{b_{1}}{\theta_{D}+1},
$$

where $b_{0}=\theta_{0}>\theta_{1}>\cdots>\theta_{D}$ are the $D+1$ distinct eigenvalues of $\Gamma$.

For the above-mentioned intersection array, $T_{\Gamma}(x)$ is a polynomial of degree 4 with a negative leading term and the following roots: $-\frac{7}{3}\left(=-1-\frac{b_{1}}{\theta_{1}+1}\right), \frac{9-\sqrt{249}}{4} \approx-1.695, \frac{17}{3}$ $\left(=-1-\frac{b_{1}}{\theta_{D}+1}\right), \frac{9+\sqrt{249}}{9} \approx 6.195$.

Thus, combining (4.1) and (4.2), we obtain

$$
-\frac{7}{3} \leq \eta \leq \frac{9-\sqrt{249}}{4} \text { or } \eta=\frac{17}{3},
$$

and one can finally obtain a contradiction as in [18, Claim 4.3].

\subsection{Infinite families}

The data from Tables 1, 2 and 3 allows us to look for infinite families of Krein arrays for which we can show nonexistence of corresponding $Q$-polynomial association schemes. We find three families, one for each number of classes. 
The first family of Krein arrays is given by

$$
\left\{2 r^{2}-1,2 r^{2}-2, r^{2}+1 ; 1,2, r^{2}-1\right\} .
$$

This Krein array is feasible for all integers $r \geq 2$. A $Q$-polynomial association scheme with Krein array (4.3) has 3 classes and $4 r^{4}$ vertices. Examples exist when $r$ is a power of 2 - they are realized by duals of Kasami codes with minimum distance 5 , see [5, §11.2].

Theorem 4.9. A Q-polynomial association scheme with Krein array (4.3) and $r$ odd does not exist.

Proof. Consider a $Q$-polynomial association scheme with Krein array (4.3). Besides the Krein parameters failing the triangle inequality, $q_{11}^{1}$ is also zero. Therefore, in order to compute triple intersection numbers, the system of equations (2.3) can be augmented with four equations derived from Theorem 2.1. We compute triple intersection numbers with respect to vertices $x, y, z$ such that $(x, y),(x, z) \in R_{1}$ and $(y, z) \in R_{2}$. Since $p_{11}^{2}=$ $r(r+2)\left(r^{2}-1\right) / 4>0$, such vertices must exist. We obtain a four parameter solution (see the notebook $Q P \circ I_{Y}-d 3-1$ param-odd. ipynb on the sage-drg package repository for computation details). Then we may express

$$
\left[\begin{array}{lll}
1 & 2 & 3
\end{array}\right]=-\frac{r^{4}}{2}+2 r^{2}+\left[\begin{array}{lll}
1 & 3 & 1
\end{array}\right]+3 \cdot\left[\begin{array}{lll}
2 & 3 & 3
\end{array}\right]-\left[\begin{array}{lll}
3 & 1 & 1
\end{array}\right]+4 \cdot\left[\begin{array}{lll}
3 & 3 & 3
\end{array}\right] .
$$

Clearly, the above triple intersection number can only be integral when $r$ is even. Therefore, we conclude that a $Q$-polynomial association scheme with Krein array (4.3) and $r$ odd does not exist.

The next family is a two parameter family of Krein arrays

$$
\left\{m, m-1, m\left(r^{2}-1\right) / r^{2}, m-r^{2}+1 ; 1, m / r^{2}, r^{2}-1, m\right\} .
$$

This Krein array is feasible for all integers $m$ and $r$ such that $0<2\left(r^{2}-1\right) \leq m \leq$ $r(r-1)(r+2)$ and $m(r+1)$ is even. A $Q$-polynomial association scheme with Krein array (4.4) is $Q$-bipartite with 4 classes and $2 m^{2}$ vertices. One may take the $Q$-bipartite quotient of such a scheme (i.e., identify vertices in relation $R_{4}$ ) to obtain a strongly regular graph with parameters $(n, k, \lambda, \mu)=\left(m^{2},(m-1) r^{2}, m+r^{2}\left(r^{2}-3\right), r^{2}\left(r^{2}-1\right)\right)$, i.e., a pseudo-Latin square graph. Therefore, we say that a scheme with Krein array (4.4) is of Latin square type.

There are several examples of $Q$-polynomial association schemes with Krein array (4.4) for some $r$ and $m$. For $(r, m)=(2,6)$ and $(r, m)=(3,16)$, this Krein array is realized by the schemes of shortest vectors of the $E_{6}$ lattice and an overlattice of the Barnes-Wall lattice in $\mathbb{R}^{16}$ [28], respectively. For $(r, m)=\left(2^{i j}, 2^{i(2 j+1)}\right)$, there are examples arising from duals of extended Kasami codes [5, §11.2] for each choice of positive integers $i$ and $j$. In particular, the Krein array obtained by setting $i=j=1$ uniquely determines the halved 8-cube.

In the case when $r$ is a prime power and $m=r^{3}$, the formal dual of this parameter set (i.e., a distance-regular graph with the corresponding intersection array) is realized by a Pasechnik graph [6].

Theorem 4.10. A Q-polynomial association scheme with Krein array (4.4) and $m$ odd does not exist. 
Proof. Consider a $Q$-polynomial association scheme with Krein array (4.4). Since the scheme is $Q$-bipartite, we have $q_{i j}^{k}=0$ whenever $i+j+k$ is odd or the triple $(i, j, k)$ does not satisfy the triangle inequality. This allows us to augment the system of equations (2.3) with many equations derived from Theorem 2.1. We compute triple intersection numbers with respect to vertices $x, y, z$ such that $(x, y),(x, z) \in R_{1}$ and $(y, z) \in R_{2}$. Since $p_{11}^{2}=$ $r^{2}\left(r^{2}-1\right) / 2>0$, such vertices must exist. We obtain a one parameter solution (see the notebook $Q P \circ I_{Y}-d 4-L S-o d d$. ipynb on the sage-drg package repository for computation details) which allows us to express

$$
\left[\begin{array}{lll}
1 & 1 & 3
\end{array}\right]=r+\frac{r^{2}(1-r)}{2}-\frac{m}{2}+\left[\begin{array}{lll}
1 & 1 & 1
\end{array}\right] .
$$

Clearly, the above triple intersection number can only be integral when $m$ is even. Therefore, we conclude that a $Q$-polynomial association scheme with Krein array (4.4) and $m$ odd does not exist.

The last family is given by the Krein array

$$
\begin{aligned}
&\left\{\frac{r^{2}+1}{2}, \frac{r^{2}-1}{2}, \frac{\left(r^{2}+1\right)^{2}}{2 r(r+1)}, \frac{(r-1)\left(r^{2}+1\right)}{4 r}, \frac{r^{2}+1}{2 r} ;\right. \\
&\left.1, \frac{(r-1)\left(r^{2}+1\right)}{2 r(r+1)}, \frac{(r+1)\left(r^{2}+1\right)}{4 r}, \frac{(r-1)\left(r^{2}+1\right)}{2 r}, \frac{r^{2}+1}{2}\right\} .
\end{aligned}
$$

This Krein array is feasible for all odd $r \geq 5$. A $Q$-polynomial association scheme with Krein array (4.5) is $Q$-bipartite with 5 classes and $2(r+1)\left(r^{2}+1\right)$ vertices. One may take the $Q$-bipartite quotient of such a scheme to obtain a strongly regular graph with parameters $(n, k, \lambda, \mu)=\left((r+1)\left(r^{2}+1\right), r(r+1), r-1, r+1\right)-$ these are precisely the parameters of collinearity graphs of generalized quadrangles $\mathrm{GQ}(r, r)$. The scheme also has a second $Q$-polynomial ordering of eigenspaces, namely the ordering $E_{5}, E_{2}, E_{3}, E_{4}, E_{1}$ relative to the ordering implied by the Krein array. For $r \equiv 1(\bmod 4)$ a prime power, the Krein array (4.5) is realized by a scheme derived by Moorhouse and Williford [30] from a double cover of the $C_{2}(r)$ dual polar graph.

Theorem 4.11. A Q-polynomial association scheme with Krein array (4.5) and $r \equiv 3$ $(\bmod 4)$ does not exist.

Proof. Consider a $Q$-polynomial association scheme with Krein array (4.5). Since the scheme is $Q$-bipartite, we have $q_{i j}^{k}=0$ whenever $i+j+k$ is odd or the triple $(i, j, k)$ does not satisfy the triangle inequality. This allows us to augment the system of equations (2.3) with many equations derived from Theorem 2.1. We compute triple intersection numbers with respect to vertices $x, y, z$ that are mutually in relation $R_{1}$. Since $p_{11}^{1}=(r-1) / 2>0$, such vertices must exist. We obtain a single solution (see the notebook $Q P \circ I_{y}-d 5-1$ param-3mod4. ipynb on the sage-drg package repository for computation details) with

$$
\left[\begin{array}{lll}
1 & 1 & 1
\end{array}\right]=\frac{r-5}{4}
$$

Clearly, the above triple intersection number can only be integral when $r \equiv 1(\bmod 4)$. Therefore, we conclude that a $Q$-polynomial association scheme with Krein array (4.5) and $r \equiv 3(\bmod 4)$ does not exist. 


\section{Quadruple intersection numbers}

The argument of the proof of Theorem 2.1 ([5, Theorem 2.3.2]) can be further extended to $s$-tuples of vertices (see Remark (iii) in [5, §2.3]; cf. [34, Lemma 4(2)]). In particular, we may consider quadruple intersection numbers with respect to a quadruple of vertices $w, x, y, z \in X$. For integers $h, i, j, k(0 \leq h, i, j, k \leq D)$, denote by $\left[\begin{array}{cccc}w & x & y & z \\ h & i & j & k\end{array}\right]$ (or simply $[h i j k]$ when it is clear which quadruple $(w, x, y, z)$ we have in mind) the number of vertices $u \in X$ such that $(u, w) \in R_{h},(u, x) \in R_{i},(u, y) \in R_{j}$, and $(u, z) \in R_{k}$.

For a fixed quadruple $(w, x, y, z)$, one can obtain a system of linear Diophantine equations with quadruple intersection numbers as variables which relates them to the intersection numbers (or to the triple intersection numbers).

The following analogue of Theorem 2.1 allows us to obtain some additional equations.

Theorem 5.1. Let $\left(X,\left\{R_{i}\right\}_{i=0}^{D}\right)$ be an association scheme of D classes with second eigenmatrix $Q$ and Krein parameters $q_{i j}^{k}(0 \leq i, j, k \leq D)$. Then, for fixed indices $\iota_{1}, \iota_{2}, \iota_{3}, \iota_{4}$ $\left(0 \leq \iota_{1}, \iota_{2}, \iota_{3}, \iota_{4} \leq D\right)$ and any permutation $p, r, s, t$ of $\iota_{1}, \iota_{2}, \iota_{3}, \iota_{4}$,

$$
\sum_{\ell=0}^{D} q_{p r}^{\ell} q_{s t}^{\ell}=0 \Longleftrightarrow \sum_{h, i, j, k=0}^{D} Q_{h p} Q_{i r} Q_{j s} Q_{k t}\left[\begin{array}{cccc}
w & x & y & z \\
h & i & j & k
\end{array}\right]=0 \quad \text { for all } w, x, y, z \in X .
$$

Proof. Since $E_{i}$ is a symmetric idempotent matrix, one has

$$
\sum_{w \in X} E_{i}(u, w) E_{i}(v, w)=E_{i}(u, v)
$$

Let $\Sigma(M)$ denote the sum of all entries of a matrix $M$. Then, by (5.1),

$$
\begin{aligned}
\Sigma\left(E_{p} \circ E_{r} \circ E_{s} \circ E_{t}\right)= & \sum_{u, v \in X} E_{p}(u, v) E_{r}(u, v) E_{s}(u, v) E_{t}(u, v) \\
= & \sum_{w, x, y, z \in X}\left(\sum_{u \in X} E_{p}(u, w) E_{r}(u, x) E_{s}(u, y) E_{t}(u, z)\right) . \\
& \left(\sum_{v \in X} E_{p}(v, w) E_{r}(v, x) E_{s}(v, y) E_{t}(v, z)\right) \\
= & \sum_{w, x, y, z \in X} \sigma(w, x, y, z)^{2} \geq 0
\end{aligned}
$$

where $\sigma(w, x, y, z)=\sum_{u \in X} E_{p}(u, w) E_{r}(u, x) E_{s}(u, y) E_{t}(u, z)$.

On the other hand, by (2.1),

$$
\begin{aligned}
|X|^{2} \Sigma\left(E_{p} \circ E_{r} \circ E_{s} \circ E_{t}\right) & =|X|^{2} \operatorname{Tr}\left(\left(E_{p} \circ E_{r}\right) \cdot\left(E_{s} \circ E_{t}\right)\right) \\
& =\operatorname{Tr}\left(\left(\sum_{\ell=0}^{D} q_{p r}^{\ell} E_{\ell}\right) \cdot\left(\sum_{\ell=0}^{D} q_{s t}^{\ell} E_{\ell}\right)\right) \\
& =\sum_{\ell=0}^{D} m_{\ell} q_{p r}^{\ell} q_{s t}^{\ell},
\end{aligned}
$$


where $m_{\ell}$ is the rank of $E_{\ell}$ (i.e., the multiplicity of the corresponding eigenspace), and by (2.2),

$$
\begin{aligned}
|X|^{3} \Sigma\left(E_{p} \circ E_{r} \circ E_{s} \circ E_{t}\right) & =\frac{1}{|X|} \sum_{\ell=0}^{D} Q_{\ell p} Q_{\ell r} Q_{\ell s} Q_{\ell t} \Sigma\left(A_{\ell}\right) \\
& =\sum_{\ell=0}^{D} n_{\ell} Q_{\ell p} Q_{\ell r} Q_{\ell s} Q_{\ell t},
\end{aligned}
$$

where $n_{\ell}$ is the valency of $\left(X, R_{\ell}\right)$.

Since the multiplicities $m_{\ell}$ are positive numbers and the Krein parameters are nonnegative numbers, by (5.2), (5.3), (5.4), we have $\Sigma\left(E_{p} \circ E_{r} \circ E_{s} \circ E_{t}\right)=0$ if and only if $q_{p r}^{\ell} q_{s t}^{\ell}=0$ (with fixed $\left.p, r, s, t\right)$ for all $\ell=0, \ldots, D$. In this case, we have $\sigma(w, x, y, z)=0$ for all quadruples $(w, x, y, z)$, which implies

$$
\begin{aligned}
0=|X|^{4} \sigma(w, x, y, z) & =|X|^{4} \sum_{u \in X} E_{p}(u, w) E_{r}(u, x) E_{s}(u, y) E_{t}(u, z) \\
& =\sum_{h, i, j, k=0}^{D} Q_{h p} Q_{i r} Q_{j s} Q_{k t}\left[\begin{array}{cccc}
w & x & y & z \\
h & i & j & k
\end{array}\right]
\end{aligned}
$$

which completes the proof.

The condition of Theorem 5.1 is satisfied when, for example, an association scheme is $Q$-bipartite, i.e., $q_{i j}^{k}=0$ whenever $i+j+k$ is odd (take $p+r$ and $s+t$ of different parity).

Suda [33] lists several families of association schemes which are known to be triply regular, i.e., their triple intersection numbers $\left[\begin{array}{ccc}x & y & z \\ i & j & k\end{array}\right]$ only depend on $i, j, k$ and the mutual distances between $x, y, z$, and not on the choices of the vertices themselves:

- strongly regular graphs with $q_{11}^{1}=0$ (cf. [8]),

- Taylor graphs (antipodal $Q$-bipartite schemes of 3 classes),

- linked systems of symmetric designs (certain $Q$-antipodal schemes of 3 classes) with $a_{1}^{*}=0$,

- tight spherical 7-designs (certain $Q$-bipartite schemes of 4 classes), and

- collections of real mutually unbiased bases $(Q$-antipodal $Q$-bipartite schemes of 4 classes).

Schemes belonging to the above families seem natural candidates for the computations of their quadruple intersection numbers. However, the condition of Theorem 5.1 is never satisfied for primitive strongly regular graphs, while for Taylor graphs the obtained equations do not give any information that could not be obtained through relating the quadruple intersection numbers to the triple intersection numbers. This was also the case for the examples of triply regular linked systems of symmetric designs that we have checked. However, in the cases of tight spherical 7-designs and mutually unbiased bases, we do get new restrictions on quadruple intersection numbers. So far, we have not succeeded in using this new information for either new constructions or proofs of nonexistence. 


\section{ORCID iDs}

Alexander L. Gavrilyuk (D) https://orcid.org/0000-0001-9296-0313

Janoš Vidali (D) https://orcid.org/0000-0001-8061-9169

Jason S. Williford (D) https://orcid.org/0000-0002-8697-5997

\section{References}

[1] E. Bannai and T. Ito, Algebraic Combinatorics I: Association Schemes, The Benjamin/Cummings Publishing, Menlo Park, CA, 1984.

[2] W. Bosma, J. Cannon and C. Playoust, The Magma algebra system I: The user language, J. Symbolic Comput. 24 (1997), 235-265, doi:10.1006/jsco.1996.0125.

[3] A. E. Brouwer, Parameters of distance-regular graphs, 2011, http://www . win. tue.nl/ aeb/drg/drgtables.html.

[4] A. E. Brouwer, Strongly regular graphs, 2013, http://www.win.tue.nl/ aeb/ graphs/srg/srgtab.html.

[5] A. E. Brouwer, A. M. Cohen and A. Neumaier, Distance-Regular Graphs, volume 18 of Ergebnisse der Mathematik und ihrer Grenzgebiete, Springer-Verlag, Berlin, 1989, doi: 10.1007/978-3-642-74341-2.

[6] A. E. Brouwer and D. V. Pasechnik, Two distance-regular graphs, J. Algebraic Combin. 36 (2012), 403-407, doi:10.1007/s10801-011-0341-1.

[7] P. J. Cameron, J.-M. Goethals and J. J. Seidel, The Krein condition, spherical designs, Norton algebras and permutation groups, Nederl. Akad. Wetensch. Indag. Math. 40 (1978), 196-206, doi:10.1016/1385-7258(78)90037-9.

[8] P. J. Cameron, J.-M. Goethals and J. J. Seidel, Strongly regular graphs having strongly regular subconstituents, J. Algebra 55 (1978), 257-280, doi:10.1016/0021-8693(78)90220-X.

[9] D. R. Cerzo and H. Suzuki, Non-existence of imprimitive $Q$-polynomial schemes of exceptional type with $d=4$, European J. Combin. 30 (2009), 674-681, doi:10.1016/j.ejc.2008.07. 014.

[10] K. Coolsaet and A. Jurišić, Using equality in the Krein conditions to prove nonexistence of certain distance-regular graphs, J. Comb. Theory Ser. A 115 (2008), 1086-1095, doi:10.1016/j. jcta.2007.12.001.

[11] E. van Dam, W. Martin and M. Muzychuk, Uniformity in association schemes and coherent configurations: cometric $Q$-antipodal schemes and linked systems, J. Comb. Theory Ser. A 120 (2013), 1401-1439, doi:10.1016/j.jcta.2013.04.004.

[12] E. R. van Dam, Three-class association schemes, J. Algebraic Combin. 10 (1999), 69-107, doi:10.1023/a:1018628204156.

[13] E. R. van Dam, J. H. Koolen and H. Tanaka, Distance-regular graphs, Electron. J. Combin. (2016), \#DS22, doi:10.37236/4925.

[14] E. R. van Dam and M. Muzychuk, Some implications on amorphic association schemes, $J$. Comb. Theory Ser. A 117 (2010), 111-127, doi:10.1016/j.jcta.2009.03.018.

[15] P. Delsarte, An algebraic approach to the association schemes of coding theory, Philips Res. Rep. Suppl. 10, Philips Research Laboratories, 1973.

[16] J. Forrest, T. Ralphs, S. Vigerske, L. Hafer, B. Kristjansson, J. P. Fasano, E. Straver, M. Lubin, H. G. Santos, R. Lougee and M. Saltzman, coin-or/Cbc (COIN-OR Branch-andCut MIP Solver), Version 2.9.4, 2015, doi:10.5281/zenodo.1317566, https : / projects. coin-or.org/Cbc. 
[17] A. L. Gavrilyuk and J. H. Koolen, The Terwilliger polynomial of a $Q$-polynomial distanceregular graph and its application to pseudo-partition graphs, Linear Algebra Appl. 466 (2015), 117-140, doi:10.1016/j.laa.2014.09.048.

[18] A. L. Gavrilyuk and J. H. Koolen, A characterization of the graphs of bilinear $(d \times d)$-forms over $\mathbb{F}_{2}$, Combinatorica 39 (2019), 289-321, doi:10.1007/s00493-017-3573-4.

[19] T. Ikuta, T. Ito and A. Munemasa, On pseudo-automorphisms and fusions of an association scheme, European J. Combin. 12 (1991), 317-325, doi:10.1016/s0195-6698(13)80114-x.

[20] T. Ito, A. Munemasa and M. Yamada, Amorphous association schemes over the Galois rings of characteristic 4, European J. Combin. 12 (1991), 513-526, doi:10.1016/s0195-6698(13) 80102-3.

[21] A. Jurišić, J. Koolen and P. Terwilliger, Tight distance-regular graphs, J. Algebraic Combin. 12 (2000), 163-197, doi:10.1023/a:1026544111089.

[22] A. Jurišić and J. Vidali, Extremal 1-codes in distance-regular graphs of diameter 3, Des. Codes Cryptogr. 65 (2012), 29-47, doi:10.1007/s10623-012-9651-0.

[23] A. Jurišić and J. Vidali, Restrictions on classical distance-regular graphs, J. Algebraic Combin. 46 (2017), 571-588, doi:10.1007/s10801-017-0765-3.

[24] B. G. Kodalen, Cometric Association Schemes, Ph.D. thesis, Worcester Polytechnic Institute, 2019, arXiv:1905.06959 [math.CO].

[25] B. G. Kodalen, Linked systems of symmetric designs, Algebr. Comb. 2 (2019), 119-147, doi: 10.5802/alco.22.

[26] A. Makhorin, GLPK (GNU Linear Programming Kit), Version 4.63.p2, 2012, http: / /www . gnu.org/software/glpk/.

[27] W. J. Martin, M. Muzychuk and J. Williford, Imprimitive cometric association schemes: constructions and analysis, J. Algebraic Combin. 25 (2007), 399-415, doi:10.1007/ s10801-006-0043-2.

[28] W. J. Martin and H. Tanaka, Commutative association schemes, European J. Combin. 30 (2009), 1497-1525, doi:10.1016/j.ejc.2008.11.001.

[29] W. J. Martin and J. Williford, There are finitely many $Q$-polynomial association schemes with given first multiplicity at least three, European J. Combin. 30 (2009), 698-704, doi:10.1016/j. ejc.2008.07.009.

[30] G. E. Moorhouse and J. Williford, Double covers of symplectic dual polar graphs, Discrete Math. 339 (2016), 571-588, doi:10.1016/j.disc.2015.09.015.

[31] T. Penttila and J. Williford, New families of $Q$-polynomial association schemes, J. Comb. Theory Ser. A 118 (2011), 502-509, doi:10.1016/j.jcta.2010.08.001.

[32] The Sage Developers, Sagemath, the Sage Mathematics Software System (Version 7.6), 2017, http: / / www. sagemath.org.

[33] S. Suda, Coherent configurations and triply regular association schemes obtained from spherical designs, J. Comb. Theory Ser. A 117 (2010), 1178-1194, doi:10.1016/j.jcta.2010.03.016.

[34] H. Suzuki, Imprimitive Q-polynomial association schemes, J. Algebraic Combin. 7 (1998), 165-180, doi:10.1023/a:1008660421667.

[35] H. Tanaka and R. Tanaka, Nonexistence of exceptional imprimitive $Q$-polynomial association schemes with six classes, European J. Combin. 32 (2011), 155-161, doi:10.1016/j.ejc.2010.09. 006.

[36] M. Urlep, Triple intersection numbers of $Q$-polynomial distance-regular graphs, European J. Combin. 33 (2012), 1246-1252, doi:10.1016/j.ejc.2012.02.005. 
A. L. Gavrilyuk et al.: On few-class Q-polynomial association schemes: feasible ...

[37] J. Vidali, Using symbolic computation to prove nonexistence of distance-regular graphs, Electron. J. Combin. 25 (2018), \#P4.21, doi:10.37236/7763.

[38] J. Vidali, jaanos/sage-drg: sage-drg Sage Package, Version 0.9, 2019, doi:10.5281/ zenodo. 3350856 .

[39] J. S. Williford, Homepage, 2018, http: //www. uwyo.edu/jwilliford/. 


\section{Appendix A Tables of nonexistence results}

Here, we give the tables of nonexistence results obtained by running the algorithm from Subsection 4.1 on the open cases in the tables from Section 3. Tables 1, 2 and 3 give nonexistence results for $Q$-polynomial schemes which are primitive of 3 classes, and $Q$ bipartite (but not $Q$-antipodal) of 4 and 5 classes, respectively.

\begin{tabular}{|c|c|c|c|c|}
\hline Label & Krein array & DRG & Nonexistence & Family \\
\hline$\langle 91,12\rangle$ & $\left\{12, \frac{338}{35}, \frac{39}{25} ; 1, \frac{312}{175}, \frac{39}{5}\right\}$ & & $(3,3,3)$ & \\
\hline$\langle 225,24\rangle$ & $\left\{24,20, \frac{36}{11} ; 1, \frac{30}{11}, 24\right\}$ & & $(3,1,1 ; 3,3,1)$ & \\
\hline$\langle 324,17\rangle$ & $\{17,16,10 ; 1,2,8\}$ & & $(1,1,2)$ & $(4.3)$ \\
\hline$\langle 324,19\rangle$ & $\left\{19, \frac{128}{9}, 10 ; 1, \frac{16}{9}, 10\right\}$ & & $(1,1,3)$ & \\
\hline$\langle 441,20\rangle$ & $\left\{20, \frac{378}{25}, 12 ; 1, \frac{42}{25}, 9\right\}$ & & $(1,1,3)$ & \\
\hline$\langle 540,33\rangle$ & $\left\{33,20, \frac{63}{5} ; 1, \frac{12}{5}, 15\right\}$ & & $(1,1,3)$ & \\
\hline$\langle 540,35\rangle$ & $\left\{35, \frac{243}{10}, \frac{27}{2} ; 1, \frac{27}{10}, \frac{45}{2}\right\}$ & & $(1,1,3)$ & \\
\hline$\langle 576,23\rangle$ & $\left\{23, \frac{432}{25}, 15 ; 1, \frac{48}{25}, 9\right\}$ & & $(1,1,3)$ & \\
\hline$\langle 729,26\rangle$ & $\left\{26, \frac{486}{25}, 18 ; 1, \frac{54}{25}, 9\right\}$ & & $(1,1,3)$ & \\
\hline$\langle 1000,37\rangle$ & $\{37,24,14 ; 1,2,12\}$ & & $(1,1,3)$ & \\
\hline$\langle 1015,28\rangle$ & $\left\{28, \frac{2523}{130}, \frac{4263}{338} ; 1, \frac{1218}{845}, \frac{203}{26}\right\}$ & & $(1,1,3)$ & \\
\hline$\langle 1080,83\rangle$ & $\{83,54,21 ; 1,6,63\}$ & FSD & $(1,1,2)$ & \\
\hline$\langle 1134,49\rangle$ & $\left\{49,48, \frac{644}{75} ; 1, \frac{196}{75}, 42\right\}$ & & $(1,1,1)$ & \\
\hline$\langle 1189,40\rangle$ & $\left\{40, \frac{5043}{203}, \frac{123}{7} ; 1, \frac{615}{406}, \frac{164}{7}\right\}$ & & $(1,1,2)$ & \\
\hline$\langle 1470,104\rangle$ & $\{104,70,25 ; 1,7,80\}$ & FSD & $(1,1,2 ; 3,2,3)$ & \\
\hline$\langle 1548,35\rangle$ & $\left\{35, \frac{2187}{86}, \frac{45}{2} ; 1, \frac{135}{86}, \frac{27}{2}\right\}$ & & $(1,1,3)$ & \\
\hline$\langle 1680,69\rangle \mathrm{a}$ & $\{69,42,7 ; 1,2,63\}$ & & $(1,1,2)$ & \\
\hline$\langle 1702,45\rangle$ & $\left\{45, \frac{4761}{148}, \frac{115}{4} ; 1, \frac{345}{148}, \frac{69}{4}\right\}$ & & $(1,1,2)$ & \\
\hline$\langle 1944,29\rangle$ & $\{29,22,25 ; 1,2,5\}$ & & $(1,1,2)$ & \\
\hline$\langle 2016,195\rangle$ & $\{195,160,28 ; 1,20,168\}$ & FSD & $(1,2,2)$ & \\
\hline$\langle 2106,65\rangle$ & $\left\{65,64, \frac{676}{25} ; 1, \frac{104}{25}, 26\right\}$ & $\{125,108,24 ; 1,9,75\}$ & $(1,1,1)$ & \\
\hline$\langle 2185,114\rangle$ & $\left\{114, \frac{4761}{65}, \frac{58121}{1521} ; 1, \frac{11799}{1690}, \frac{6118}{117}\right\}$ & & $(1,1,3)$ & \\
\hline$\langle 2197,36\rangle$ & $\left\{36, \frac{45}{2}, \frac{45}{2} ; 1, \frac{3}{2}, \frac{15}{2}\right\}$ & & $(1,1,3)$ & \\
\hline$\langle 2197,126\rangle$ & $\{126,90,10 ; 1,6,105\}$ & FSD $(0231)$ & $(2,2,3)$ & \\
\hline$\langle 2304,47\rangle$ & $\left\{47, \frac{135}{4}, 33 ; 1, \frac{9}{4}, 15\right\}$ & & $(1,1,3)$ & \\
\hline$\langle 2376,95\rangle$ & $\{95,63,12 ; 1,3,84\}$ & & $(1,1,3)$ & \\
\hline$\langle 2401,48\rangle$ & $\left\{48,30,29 ; 1, \frac{3}{2}, 20\right\}$ & & $(1,1,2)$ & \\
\hline$\langle 2500,49\rangle \mathrm{a}$ & $\{49,48,26 ; 1,2,24\}$ & & $(1,1,2)$ & $(4.3)$ \\
\hline$\langle 2640,203\rangle$ & $\{203,160,34 ; 1,16,170\}$ & FSD & $(1,2,2)$ & \\
\hline
\end{tabular}

Table 1: Nonexistence results for feasible Krein arrays of primitive 3-class $Q$-polynomial association schemes on up to 2800 vertices. For $P$-polynomial parameters (for the natural ordering of relations, unless otherwise indicated), the DRG column indicates whether the parameters are formally self-dual (FSD), or the intersection array is given. The Nonexistence column gives either the triple of relation indices for which there is no solution for triple intersection numbers, or the 6-tuple of relation indices $(r, s, t ; i, j, k)$ for which Corollary 4.2 is not satisfied. The Family column specifies the infinite family from Subsection 4.2 that the parameter set is part of. 


\begin{tabular}{|c|c|c|c|}
\hline Label & Krein array & Nonexistence & Family \\
\hline$\overline{\langle\langle 200,12\rangle}$ & $\left\{12,11, \frac{256}{25}, \frac{36}{11} ; 1, \frac{44}{25}, \frac{96}{11}, 12\right\}$ & $(1,1,2)$ & \\
\hline$\langle 462,21\rangle$ & $\left\{21,20, \frac{196}{11}, \frac{49}{5} ; 1, \frac{35}{11}, \frac{56}{5}, 21\right\}$ & $(1,1,2)$ & \\
\hline$\langle 486,45\rangle$ & $\{45,44,36,5 ; 1,9,40,45\}$ & $(1,1,2)$ & \\
\hline$\langle 578,17\rangle$ & $\left\{17,16, \frac{136}{9}, 9 ; 1, \frac{17}{9}, 8,17\right\}$ & $(1,1,2)$ & $(4.4)$ \\
\hline$\langle 686,28\rangle$ & $\{28,27,25,8 ; 1,3,20,28\}$ & $(1,2,2)$ & \\
\hline$\langle 702,36\rangle$ & $\left\{36,35, \frac{405}{13}, \frac{72}{7} ; 1, \frac{63}{13}, \frac{180}{7}, 36\right\}$ & $(1,2,2)$ & \\
\hline$\langle 722,19\rangle$ & $\left\{19,18, \frac{152}{9}, 11 ; 1, \frac{19}{9}, 8,19\right\}$ & $(1,1,2)$ & $(4.4)$ \\
\hline$\langle 882,21\rangle$ & $\left\{21,20, \frac{56}{3}, 13 ; 1, \frac{7}{3}, 8,21\right\}$ & $(1,1,2)$ & $(4.4)$ \\
\hline$\langle 990,66\rangle$ & $\left\{66,65, \frac{847}{15}, \frac{88}{13} ; 1, \frac{143}{15}, \frac{770}{13}, 66\right\}$ & $(1,2,2)$ & \\
\hline$\langle 1014,78\rangle$ & $\{78,77,65,8 ; 1,13,70,78\}$ & $(1,2,2)$ & \\
\hline$\langle 1058,23\rangle$ & $\left\{23,22, \frac{184}{9}, 15 ; 1, \frac{23}{9}, 8,23\right\}$ & $(1,1,2)$ & $(4.4)$ \\
\hline$\langle 1250,25\rangle$ & $\left\{25,24, \frac{200}{9}, 17 ; 1, \frac{25}{9}, 8,25\right\}$ & $(1,1,2)$ & (4.4) \\
\hline$\langle 1458,27\rangle$ & $\{27,26,24,19 ; 1,3,8,27\}$ & $(1,1,2)$ & $(4.4)$ \\
\hline$\langle 1458,36\rangle$ & $\{36,35,33,16 ; 1,3,20,36\}$ & $(1,2,2)$ & \\
\hline$\langle 1482,38\rangle$ & $\left\{38,37, \frac{12635}{351}, \frac{76}{37} ; 1, \frac{703}{351}, \frac{1330}{37}, 38\right\}$ & $(1,2,2)$ & \\
\hline$\langle 1674,45\rangle$ & $\left\{45,44, \frac{1296}{31}, \frac{135}{11} ; 1, \frac{99}{31}, \frac{360}{11}, 45\right\}$ & $(1,1,2)$ & \\
\hline$\langle 1682,29\rangle$ & $\left\{29,28, \frac{232}{9}, 21 ; 1, \frac{29}{9}, 8,29\right\}$ & $(1,1,2)$ & $(4.4)$ \\
\hline$\langle 1694,55\rangle$ & $\left\{55,54, \frac{352}{7}, 15 ; 1, \frac{33}{7}, 40,55\right\}$ & $(1,1,2)$ & \\
\hline$\langle 1862,21\rangle$ & $\left\{21,20, \frac{364}{19}, \frac{81}{5} ; 1, \frac{35}{19}, \frac{24}{5}, 21\right\}$ & $(1,1,2)$ & \\
\hline$\langle 2058,49\rangle$ & $\left\{49,48, \frac{686}{15}, \frac{77}{5} ; 1, \frac{49}{15}, \frac{168}{5}, 49\right\}$ & $(1,1,2)$ & \\
\hline$\langle 2060,50\rangle$ & $\left\{50,49, \frac{4800}{103}, \frac{110}{7} ; 1, \frac{350}{103}, \frac{240}{7}, 50\right\}$ & $(1,1,2)$ & \\
\hline$\langle 2394,27\rangle$ & $\left\{27,26, \frac{3240}{133}, \frac{279}{13} ; 1, \frac{351}{133}, \frac{72}{13}, 27\right\}$ & $(1,1,2)$ & \\
\hline$\langle 2466,36\rangle$ & $\left\{36,35, \frac{4617}{137}, \frac{144}{7} ; 1, \frac{315}{137}, \frac{108}{7}, 36\right\}$ & $(1,2,2)$ & \\
\hline$\langle 2550,85\rangle$ & $\left\{85,84, \frac{1156}{15}, \frac{187}{7} ; 1, \frac{119}{15}, \frac{408}{7}, 85\right\}$ & $(1,1,2)$ & \\
\hline$\langle 2662,121\rangle$ & $\left\{121,120, \frac{5324}{49}, \frac{77}{5} ; 1, \frac{605}{49}, \frac{528}{5}, 121\right\}$ & $(1,1,2)$ & \\
\hline$\langle 2706,66\rangle$ & $\left\{66,65, \frac{2541}{41}, \frac{44}{3} ; 1, \frac{165}{41}, \frac{154}{3}, 66\right\}$ & $(1,2,2)$ & \\
\hline$\langle 2730,78\rangle$ & $\left\{78,77, \frac{507}{7}, \frac{52}{3} ; 1, \frac{39}{7}, \frac{182}{3}, 78\right\}$ & $(1,2,2)$ & \\
\hline$\langle 2750,25\rangle$ & $\left\{25,24, \frac{250}{11}, \frac{185}{9} ; 1, \frac{25}{11}, \frac{40}{9}, 25\right\}$ & $(1,1,2)$ & \\
\hline$\langle 2862,53\rangle$ & $\left\{53,52, \frac{11236}{225}, \frac{265}{13} ; 1, \frac{689}{225}, \frac{424}{13}, 53\right\}$ & $(1,1,2)$ & \\
\hline$\langle 2890,153\rangle$ & $\{153,152,136,9 ; 1,17,144,153\}$ & $(1,1,2)$ & \\
\hline$\langle 2926,171\rangle$ & $\left\{171,170, \frac{11552}{77}, \frac{171}{17} ; 1, \frac{1615}{77}, \frac{2736}{17}, 171\right\}$ & $(1,1,2)$ & \\
\hline$\langle 2970,54\rangle$ & $\left\{54,53, \frac{567}{11}, 12 ; 1, \frac{27}{11}, 42,54\right\}$ & $(1,2,2)$ & \\
\hline$\langle 3042,65\rangle$ & $\left\{65,64, \frac{182}{3}, 25 ; 1, \frac{13}{3}, 40,65\right\}$ & $(1,1,2)$ & \\
\hline$\langle 3074,106\rangle$ & $\left\{106,105, \frac{2809}{29}, \frac{212}{9} ; 1, \frac{265}{29}, \frac{742}{9}, 106\right\}$ & $(1,2,2)$ & \\
\hline$\langle 3174,184\rangle$ & $\{184,183,161,16 ; 1,23,168,184\}$ & $(1,2,2)$ & \\
\hline$\langle 3250,50\rangle$ & $\left\{50,49, \frac{625}{13}, \frac{100}{9} ; 1, \frac{25}{13}, \frac{350}{9}, 50\right\}$ & $(1,2,2)$ & \\
\hline$\langle 3402,126\rangle$ & $\left\{126,125, \frac{343}{3}, 28 ; 1, \frac{35}{3}, 98,126\right\}$ & $(1,2,2)$ & \\
\hline$\langle 3498,77\rangle$ & $\left\{77,76, \frac{3872}{53}, \frac{231}{19} ; 1, \frac{209}{53}, \frac{1232}{19}, 77\right\}$ & $(1,1,2)$ & \\
\hline$\langle 3610,133\rangle$ & $\left\{133,132, \frac{608}{5}, 21 ; 1, \frac{57}{5}, 112,133\right\}$ & $(1,1,2)$ & \\
\hline$\langle 3726,36\rangle$ & $\left\{36,35, \frac{783}{23}, 24 ; 1, \frac{45}{23}, 12,36\right\}$ & $(1,2,2)$ & \\
\hline$\langle 4070,55\rangle$ & $\left\{55,54, \frac{1936}{37}, \frac{77}{3} ; 1, \frac{99}{37}, \frac{88}{3}, 55\right\}$ & $(1,1,2)$ & \\
\hline$\langle 4250,119\rangle$ & $\left\{119,118, \frac{13872}{125}, \frac{1309}{59} ; 1, \frac{1003}{125}, \frac{5712}{59}, 119\right\}$ & $(1,1,2)$ & \\
\hline$\langle 4370,190\rangle$ & $\left\{190,189, \frac{3971}{23}, \frac{76}{7} ; 1, \frac{399}{23}, \frac{1254}{7}, 190\right\}$ & $(1,2,2)$ & \\
\hline$\langle 4410,210\rangle$ & $\{210,209,189,12 ; 1,21,198,210\}$ & $(1,2,2)$ & \\
\hline$\langle 4464,24\rangle$ & $\left\{24,23, \frac{2048}{93}, \frac{488}{23} ; 1, \frac{184}{93}, \frac{64}{23}, 24\right\}$ & $(1,1,2)$ & \\
\hline$\langle 4526,73\rangle$ & $\left\{73,72, \frac{10658}{155}, \frac{511}{15} ; 1, \frac{657}{155}, \frac{584}{15}, 73\right\}$ & $(1,1,2)$ & \\
\hline$\langle 4558,86\rangle$ & $\left\{86,85, \frac{12943}{159}, \frac{1376}{51} ; 1, \frac{731}{159}, \frac{3010}{51}, 86\right\}$ & $(1,2,2)$ & \\
\hline$\langle 4590,75\rangle$ & $\left\{75,74, \frac{1200}{17}, 35 ; 1, \frac{75}{17}, 40,75\right\}$ & $(1,1,2)$ & \\
\hline
\end{tabular}




\begin{tabular}{|c|c|c|c|}
\hline Label & Krein array & Nonexistence & Family \\
\hline$\langle 4758,117\rangle$ & $\overline{\left\{117,116, \frac{6760}{61}, \frac{273}{29} ; 1, \frac{377}{61}, \frac{3120}{29}, 117\right\}}$ & $(1,1,2)$ & \\
\hline$\langle 4802,49\rangle$ & $\left\{49,48, \frac{1176}{25}, 25 ; 1, \frac{49}{25}, 24,49\right\}$ & $(1,1,2)$ & (4.4) \\
\hline$\langle 5046,261\rangle$ & $\{261,260,232,21 ; 1,29,240,261\}$ & $(1,1,2)$ & \\
\hline$\langle 5202,51\rangle$ & $\left\{51,50, \frac{1224}{25}, 27 ; 1, \frac{51}{25}, 24,51\right\}$ & $(1,1,2)$ & (4.4) \\
\hline$\langle 5480,100\rangle$ & $\left\{100,99, \frac{12800}{137}, \frac{140}{3} ; 1, \frac{900}{137}, \frac{160}{3}, 100\right\}$ & $(1,1,2)$ & \\
\hline$\langle 5566,66\rangle$ & $\left\{66,65, \frac{1463}{23}, 24 ; 1, \frac{55}{23}, 42,66\right\}$ & $(1,2,2)$ & \\
\hline$\langle 5590,78\rangle$ & $\left\{78,77, \frac{3211}{43}, \frac{312}{11} ; 1, \frac{143}{43}, \frac{546}{11}, 78\right\}$ & $(1,2,2)$ & \\
\hline$\langle 5618,53\rangle$ & $\left\{53,52, \frac{1272}{25}, 29 ; 1, \frac{53}{25}, 24,53\right\}$ & $(1,1,2)$ & (4.4) \\
\hline$\langle 5618,106\rangle$ & $\left\{106,105, \frac{901}{9}, 36 ; 1, \frac{53}{9}, 70,106\right\}$ & $(1,2,2)$ & \\
\hline$\langle 5642,91\rangle$ & $\left\{91,90, \frac{2704}{31}, \frac{65}{3} ; 1, \frac{117}{31}, \frac{208}{3}, 91\right\}$ & $(1,1,2)$ & \\
\hline$\langle 5670,105\rangle$ & $\{105,104,98,49 ; 1,7,56,105\}$ & $(1,1,2)$ & \\
\hline$\langle 5670,105\rangle \mathrm{a}$ & $\{105,104,100,25 ; 1,5,80,105\}$ & $(1,1,2)$ & \\
\hline$\langle 6050,55\rangle$ & $\left\{55,54, \frac{264}{5}, 31 ; 1, \frac{11}{5}, 24,55\right\}$ & $(1,1,2)$ & (4.4) \\
\hline$\langle 6278,73\rangle$ & $\left\{73,72, \frac{21316}{301}, \frac{365}{21} ; 1, \frac{657}{301}, \frac{1168}{21}, 73\right\}$ & $(1,1,2)$ & \\
\hline$\langle 6358,85\rangle$ & $\left\{85,84, \frac{884}{11}, 45 ; 1, \frac{51}{11}, 40,85\right\}$ & $(1,1,2)$ & \\
\hline$\langle 6422,91\rangle$ & $\left\{91,90, \frac{1664}{19}, \frac{119}{5} ; 1, \frac{65}{19}, \frac{336}{5}, 91\right\}$ & $(1,1,2)$ & \\
\hline$\langle 6426,147\rangle$ & $\left\{147,146, \frac{2352}{17}, 35 ; 1, \frac{147}{17}, 112,147\right\}$ & $(1,1,2)$ & \\
\hline$\langle 6450,105\rangle$ & $\left\{105,104, \frac{4320}{43}, \frac{357}{13} ; 1, \frac{195}{43}, \frac{1008}{13}, 105\right\}$ & $(1,1,2)$ & \\
\hline$\langle 6498,57\rangle$ & $\left\{57,56, \frac{1368}{25}, 33 ; 1, \frac{57}{25}, 24,57\right\}$ & $(1,1,2)$ & (4.4) \\
\hline$\langle 6962,59\rangle$ & $\left\{59,58, \frac{1416}{25}, 35 ; 1, \frac{59}{25}, 24,59\right\}$ & $(1,1,2)$ & (4.4) \\
\hline$\langle 7210,103\rangle$ & $\left\{103,102, \frac{84872}{875}, \frac{927}{17} ; 1, \frac{5253}{875}, \frac{824}{17}, 103\right\}$ & $(1,1,2)$ & \\
\hline$\langle 7442,61\rangle$ & $\left\{61,60, \frac{1464}{25}, 37 ; 1, \frac{61}{25}, 24,61\right\}$ & $(1,1,2)$ & (4.4) \\
\hline$\langle 7854,66\rangle$ & $\left\{66,65, \frac{1089}{17}, \frac{88}{3} ; 1, \frac{33}{17}, \frac{110}{3}, 66\right\}$ & $(1,2,2)$ & \\
\hline$\langle 7878,78\rangle$ & $\left\{78,77, \frac{7605}{101}, \frac{104}{3} ; 1, \frac{273}{101}, \frac{130}{3}, 78\right\}$ & $(1,2,2)$ & \\
\hline$\langle 7906,134\rangle$ & $\left\{134,133, \frac{22445}{177}, \frac{2948}{57} ; 1, \frac{1273}{177}, \frac{4690}{57}, 134\right\}$ & $(1,2,2)$ & \\
\hline$\langle 7938,63\rangle$ & $\left\{63,62, \frac{1512}{25}, 39 ; 1, \frac{63}{25}, 24,63\right\}$ & $(1,1,2)$ & (4.4) \\
\hline$\langle 8120,100\rangle$ & $\left\{100,99, \frac{19200}{203}, \frac{620}{11} ; 1, \frac{1100}{203}, \frac{480}{11}, 100\right\}$ & $(1,1,2)$ & \\
\hline$\langle 8190,90\rangle$ & $\left\{90,89, \frac{1125}{13}, 40 ; 1, \frac{45}{13}, 50,90\right\}$ & $(1,2,2)$ & \\
\hline$\langle 8246,217\rangle$ & $\left\{217,216, \frac{3844}{19}, \frac{155}{3} ; 1, \frac{279}{19}, \frac{496}{3}, 217\right\}$ & $(1,1,2)$ & \\
\hline$\langle 8450,65\rangle$ & $\left\{65,64, \frac{312}{5}, 41 ; 1, \frac{13}{5}, 24,65\right\}$ & $(1,1,2)$ & $(4.4)$ \\
\hline$\langle 8450,78\rangle$ & $\left\{78,77, \frac{377}{5}, 36 ; 1, \frac{13}{5}, 42,78\right\}$ & $(1,2,2)$ & \\
\hline$\langle 8470,88\rangle$ & $\left\{88,87, \frac{429}{5}, 16 ; 1, \frac{11}{5}, 72,88\right\}$ & $(1,2,2)$ & \\
\hline$\langle 8478,27\rangle$ & $\left\{27,26, \frac{3888}{157}, \frac{327}{13} ; 1, \frac{351}{157}, \frac{24}{13}, 27\right\}$ & $(1,1,2)$ & \\
\hline$\langle 8750,325\rangle$ & $\{325,324,300,13 ; 1,25,312,325\}$ & $(1,1,2)$ & \\
\hline$\langle 8758,232\rangle$ & $\left\{232,231, \frac{32799}{151}, \frac{464}{11} ; 1, \frac{2233}{151}, \frac{2088}{11}, 232\right\}$ & $(1,2,2)$ & \\
\hline$\langle 8798,106\rangle$ & $\left\{106,105, \frac{8427}{83}, \frac{424}{9} ; 1, \frac{371}{83}, \frac{530}{9}, 106\right\}$ & $(1,2,2)$ & \\
\hline$\langle 8802,351\rangle$ & $\left\{351,350, \frac{52488}{163}, \frac{351}{25} ; 1, \frac{4725}{163}, \frac{8424}{25}, 351\right\}$ & $(1,1,2)$ & \\
\hline$\langle 8978,67\rangle$ & $\left\{67,66, \frac{1608}{25}, 43 ; 1, \frac{67}{25}, 24,67\right\}$ & $(1,1,2)$ & (4.4) \\
\hline$\langle 9310,105\rangle$ & $\left\{105,104, \frac{17500}{171}, \frac{165}{13} ; 1, \frac{455}{171}, \frac{1200}{13}, 105\right\}$ & $(1,1,2)$ & \\
\hline$\langle 9350,153\rangle$ & $\left\{153,152, \frac{8092}{55}, \frac{459}{19} ; 1, \frac{323}{55}, \frac{2448}{19}, 153\right\}$ & $(1,1,2)$ & \\
\hline$\langle 9386,171\rangle$ & $\left\{171,170, \frac{2128}{13}, 27 ; 1, \frac{95}{13}, 144,171\right\}$ & $(1,1,2)$ & \\
\hline$\langle 9522,69\rangle$ & $\left\{69,68, \frac{1656}{25}, 45 ; 1, \frac{69}{25}, 24,69\right\}$ & $(1,1,2)$ & (4.4) \\
\hline$\langle 9522,161\rangle$ & $\left\{161,160, \frac{460}{3}, 49 ; 1, \frac{23}{3}, 112,161\right\}$ & $(1,1,2)$ & \\
\hline$\langle 9702,126\rangle$ & $\left\{126,125, \frac{1323}{11}, 56 ; 1, \frac{63}{11}, 70,126\right\}$ & $(1,2,2)$ & \\
\hline
\end{tabular}

Table 2: Nonexistence results for feasible Krein arrays of $Q$-bipartite (but not $Q$-antipodal) 4 -class $Q$-polynomial association schemes on up to 10000 vertices. The Nonexistence column gives either the triple of relation indices for which there is no solution for triple intersection numbers. The Family column specifies the infinite family from Subsection 4.2 that the parameter set is part of. 


\begin{tabular}{ccc} 
Label & Krein array & Family \\
\hline \hline$\langle 576,21\rangle$ & $\left\{21,20,18, \frac{21}{2}, \frac{27}{7} ; 1,3, \frac{21}{2}, \frac{120}{7}, 21\right\}$ \\
$\langle 800,25\rangle$ & $\left\{25,24, \frac{625}{28}, \frac{75}{7}, \frac{25}{7} ; 1, \frac{75}{28}, \frac{100}{7}, \frac{150}{7}, 25\right\}$ & $(4.5)$ \\
$\langle 2000,25\rangle$ & $\left\{25,24, \frac{625}{27}, \frac{50}{3}, \frac{25}{9} ; 1, \frac{50}{27}, \frac{25}{3}, \frac{200}{9}, 25\right\}$ \\
$\langle 2400,22\rangle$ & $\left\{22,21,20, \frac{88}{5}, \frac{32}{11} ; 1,2, \frac{22}{5}, \frac{210}{11}, 22\right\}$ \\
$\langle 2928,61\rangle$ & $\left\{61,60, \frac{3721}{66}, \frac{305}{11}, \frac{61}{11} ; 1, \frac{305}{66}, \frac{366}{11}, \frac{610}{11}, 61\right\}$ \\
$\langle 7232,113\rangle$ & $\left\{113,112, \frac{12769}{120}, \frac{791}{15}, \frac{113}{15} ; 1, \frac{791}{120}, \frac{904}{15}, \frac{1582}{15}, 113\right\}$ & $(4.5)$ \\
$\langle 14480,181\rangle$ & $\left\{181,180, \frac{32761}{190}, \frac{1629}{19}, \frac{181}{19} ; 1, \frac{1629}{190}, \frac{1810}{19}, \frac{3258}{19}, 181\right\}$ & $(4.5)$ \\
$\langle 25440,265\rangle$ & $\left\{265,264, \frac{70225}{276}, \frac{2915}{23}, \frac{265}{23} ; 1, \frac{2915}{276}, \frac{3180}{23}, \frac{5830}{23}, 265\right\}$ & $(4.5)$ \\
$\langle 37752,121\rangle$ & $\left\{121,120, \frac{14641}{125}, \frac{484}{5}, \frac{121}{25} ; 1, \frac{484}{125}, \frac{121}{5}, \frac{2904}{25}, 121\right\}$ \\
$\langle 40880,365\rangle$ & $\left\{365,364, \frac{133225}{378}, \frac{4745}{27}, \frac{365}{27} ; 1, \frac{4745}{378}, \frac{5110}{27}, \frac{9490}{27}, 365\right\}$ & \\
$\langle 47040,116\rangle$ & $\left\{116,115,112, \frac{696}{7}, \frac{144}{29} ; 1,4, \frac{116}{7}, \frac{3220}{29}, 116\right\}$
\end{tabular}

Table 3: Nonexistence results for feasible Krein arrays of $Q$-bipartite (but not $Q$-antipodal) 5 -class $Q$-polynomial association schemes on up to 50000 vertices. In all cases, there is no solution for triple intersection numbers for a triple of vertices mutually in relation $R_{1}$. The Family column specifies the infinite family from Subsection 4.2 that the parameter set is part of. 